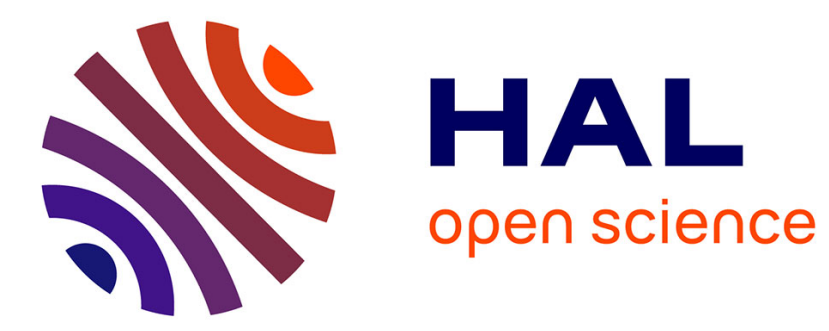

\title{
Joint Carrier Frequency Offset and Channel Estimation for OFDM Systems via the EM Algorithm in the Presence of Very High Mobility
}

Eric Pierre Simon, Laurent Ros, Hussein Hijazi, Mounir Ghogho

\section{To cite this version:}

Eric Pierre Simon, Laurent Ros, Hussein Hijazi, Mounir Ghogho. Joint Carrier Frequency Offset and Channel Estimation for OFDM Systems via the EM Algorithm in the Presence of Very High Mobility. IEEE Transactions on Signal Processing, 2012, 60 (2), pp.754-765. hal-00660684

\section{HAL Id: hal-00660684 https://hal.science/hal-00660684}

Submitted on 17 Jan 2012

HAL is a multi-disciplinary open access archive for the deposit and dissemination of scientific research documents, whether they are published or not. The documents may come from teaching and research institutions in France or abroad, or from public or private research centers.
L'archive ouverte pluridisciplinaire HAL, est destinée au dépôt et à la diffusion de documents scientifiques de niveau recherche, publiés ou non, émanant des établissements d'enseignement et de recherche français ou étrangers, des laboratoires publics ou privés. 


\title{
Joint Carrier Frequency Offset and Channel Estimation for OFDM Systems via the EM Algorithm in the presence of very high mobility
}

\author{
Eric Pierre Simon ${ }^{1}$, Laurent Ros $^{2}$, Hussein Hijazi ${ }^{3}$, Mounir Ghogho ${ }^{4}$ \\ ${ }^{1}$ IEMN lab, TELICE group, University of Lille, FRANCE \\ ${ }^{2}$ GIPSA-lab, Department Image Signal, BP 46 - 38402 Saint Martin d'Hères, FRANCE \\ ${ }^{3}$ School of Engineering, CCE Department, Lebanese International University (LIU), Lebanon \\ ${ }^{4}$ School of Electronic and Electrical Engineering, Leeds University, ENGLAND \\ e-mail: eric.simon@univ-lille1.fr, laurent.ros@gipsa-lab.grenoble-inp.fr, hussein.hijazi@liu.edu.lb, m.ghogho@leeds.ac.uk
}

\begin{abstract}
In this paper, the problem of joint Carrier Frequency Offset (CFO) and channel estimation for OFDM systems over the fast time-varying frequency-selective channel is explored within the framework of the expectation-maximization (EM) algorithm and parametric channel model. Assuming that the path delays are known, a novel iterative pilot-aided algorithm for joint estimation of the multi-path Rayleigh channel Complex Gains (CG) and the Carrier Frequency Offset (CFO) is introduced. Each CG time-variation, within one OFDM symbol, is approximated by a Basis Expansion Model (BEM) representation. An auto-regressive (AR) model is built to statistically characterize the variations of the BEM coefficients across the OFDM blocks. In addition to the algorithm, the derivation of the Hybrid Cramér-Rao Bound (HCRB) for CFO and CGs estimation in our context of very high mobility is provided. We show that the proposed EM has a lower computational complexity than the optimum maximum a posteriori estimator and yet incurs only an insignificant loss in performance.
\end{abstract}

\section{INTRODUCTION}

$\mathbf{O}$ RTHOGONAL frequency division multiplexing (OFDM) has become a standard technique for broadband high speed communication systems, mainly the Mobile Worldwide Interoperability Microwave Systems for Next-Generation Wireless Communication Systems (WiMAX) and the Third-Generation Partnership Project (3GPP) in the form of its Long-Term Evolution (LTE) project. However, it is well known that small carrier frequency offsets (CFOs) yield severe degradation in OFDM modulation since it produces inter-carrier interference (ICI) and attenuates the desired signal. These effects reduce the effective signal-to-noise ratio (SNR) in OFDM reception resulting in degraded system performance [1] [2]. Accurate CFO recovery is thus essential to make an OFDM system practically viable. In addition, the coherent detection of signals is generally performed by means of an equalization task which requires the channel knowledge. Therefore the channel estimation is also essential for practical systems.

The best performance is obtained when the CFO and the channel are estimated jointly [3]. This problem can be regarded as an estimation problem of one given parameter (here the CFO) in the presence of unobserved state (here CGs of the channel). Then, an interesting approach is to use the
Expectation-Maximization (EM) algorithm. The EM algorithm provides actually an iterative method to find the maximum likelihood (ML) estimates, as presented in [4] for the problem of speech recognition system parameters estimation while also providing the state estimates. Recently, the EM algorithm has been applied to a lot of problems including channel estimation [5] [6][7][8]. In [6], the authors proposed a joint CFO and channel estimator based on EM algorithm for OFDM - single input-single output (SISO) systems, and in [7] the authors extended this work to the OFDM - multiple input-multiple output (MIMO) context. However, these studies have been derived for quasi-static channels. We propose to address the problem of joint $\mathrm{CFO}$ and channel estimation via the EM algorithm in the context of OFDM systems with very high mobility. Note that there are other approaches for estimating both CFO and channel, like the extended Kalman filter used in [9].

In the context of very high mobility, it is generally preferable to directly estimate the physical channel parameters [10]. Indeed, as the channel delay spread increases, the number of channel taps also increases, thus leading to a large number of BEM coefficients, and consequently more pilot symbols are needed. Estimating the physical propagation parameters means estimating multi-path delays and multi-path CGs. It is well known that in Radio-Frequency transmissions, the path delays are quasi-invariant over several OFDM blocks [11](Sec.IIIA), [12], [13] (whereas the CGs may change significantly, even within one OFDM block). In this work, the delays are assumed perfectly estimated and quasi-invariant. It should be noted that an initial, and generally accurate estimation of the number of paths and delays can be obtained by using the MDL (minimum description length) and ESPRIT (estimation of signal parameters by rotational invariance techniques) methods, respectively [14][10][15]. The CFO and channel estimator derivations are presented for this approach. Additionally, we demonstrate that the results for the non-parametric approach can be simply deduced from those derivations. Hence, this work can be applied to both approaches.

The Cramér-Rao bound (CRB) is a well known benchmark to which the mean-square errors of estimators are compared. In [16], the authors derived the CRB for the joint estimation 
of CFO and channel response in a static context. In [17], the authors derived the CRB of path CGs in the presence of high mobility. We propose to extend this bound to the case of joint $\mathrm{CFO}$ and CGs estimation. Depending on the prior knowledge available on parameters, the CRB has different expressions. The hybrid CRB (HCRB) is considered in the case of a hybrid vector containing deterministic and random parameters. We provide the derivation of the HCRB for joint estimation of $\mathrm{CFO}$ and the fast time-varying CGs in the OFDM context. To sum up, the main contributions for the work presented in this paper are the following:

- Unlike existing methods, the proposed algorithm uses a parametric channel model to estimate the CFO and the channel in a highly mobile environment. Using parametric channel models is of interest in the case of high mobility, since less coefficients are to be estimated.

- To reduce estimation complexity, the proposed method uses the BEM approach (to reduce the number of coefficients to estimate), a multivariate AR(1) model (to describe the statistical variations of the BEM coefficients across the blocks), and the EM method to avoid batch processing. Simulations have shown that the proposed algorithm has almost the same performance as the optimum maximum a posteriori (MAP) estimator which requires handling and inverting large matrices.

- A closed-form expression of the HCRB is provided.

The notations adopted are as follows: Upper (lower) bold face letters denote matrices (column vectors). $[\mathbf{x}]_{n}$ denotes the $n$th element of the vector $\mathbf{x}$, and $[\mathbf{X}]_{n, m}$ denotes the $[n, m]$ th element of the matrix $\mathbf{X}$. It is noteworthy that vector and matrix indices start from 0 and not from 1. We will use the matlab notation $\mathbf{X}_{\left[n_{1}: n_{2}, m_{1}: m_{2}\right]}$ to extract a submatrix within $\mathbf{X}$ from row $n_{1}$ to row $n_{2}$ and from column $m_{1}$ to column $m_{2}$. $\mathbf{I}_{N}$ is a $N \times N$ identity matrix and $\mathbf{0}_{N}$ is a $N \times N$ matrix of zeros. $\operatorname{diag}\{\mathbf{x}\}$ is a diagonal matrix with $\mathbf{x}$ on its main diagonal, $\operatorname{diag}\{\mathbf{X}\}$ is a vector whose elements are the elements of the main diagonal of $\mathbf{X}$ and blkdiag $\{\mathbf{X}, \mathbf{Y}\}$ is a block diagonal matrix with the matrices $\mathbf{X}$ and $\mathbf{Y}$ on its main diagonal. The Hadamard product for two matrices $\mathbf{X}$ and $\mathbf{Y}$ of the same dimensions is denoted $\mathbf{X} \circ \mathbf{Y}$. The superscripts $(\cdot)^{T}$ and $(\cdot)^{H}$ stand respectively for transpose and Hermitian operators. $|\cdot|$, and $\operatorname{Tr}\{\cdot\}$ are respectively the determinant and trace operations. $\operatorname{Re}(\cdot), \operatorname{Im}(\cdot)$ and $(\cdot)^{*}$ are respectively the real part, imaginary part and conjugate of a complex number or matrix. $\mathbb{E}_{x, y}[\cdot]$ is the expectation over $x$ and $y, J_{0}(\cdot)$ is the zeroth-order Bessel function of the first kind and $\delta_{k, m}$ is the Kronecker symbol. $\nabla_{\mathbf{x}}$ and $\Delta_{\mathbf{y}}^{\mathbf{x}}$ represent the first and the second-order partial derivatives operator i.e., $\nabla_{\mathbf{x}}=\left[\frac{\partial}{\partial x_{1}}, \ldots, \frac{\partial}{\partial x_{N}}\right]^{T}$ and $\Delta_{\mathbf{y}}^{\mathbf{x}}=\nabla_{\mathbf{y}^{*}} \nabla_{\mathbf{x}}^{T}$.

\section{TIME-VARYING OFDM SYSTEM SUBJECT TO CFO}

\section{A. OFDM System Model}

Consider an OFDM system with $N$ sub-carriers, and a cyclic prefix length $N_{g}$. The duration of an OFDM block is $T=N_{b} T_{s}$, where $T_{s}$ is the sampling time and $N_{b}=N+N_{g}$. Let $x_{k}[n], n=-\frac{N}{2}, \ldots, \frac{N}{2}-1$ be the transmitted data symbol on the subcarrier $n$ of the $k$ th OFDM symbol. The $\left\{x_{k}[n]\right\}$ are normalized symbols (i.e., $\mathbb{E}\left[x_{k}[n] x_{k}^{*}[n]\right]=1$ ). The frequency mismatch between the oscillators used in the radio transmitters and receivers causes a CFO $\Delta F$. The normalized $\mathrm{CFO}$ is denoted $\nu=\Delta F N T_{s}$. After transmission over a multi-path Rayleigh channel, the subcarrier $n$ of the $k$ th received OFDM symbol $y_{k}[n]$ is given in the frequency domain (after removing cyclic prefix and taking DFT) by [13] [18]:

$$
\mathbf{y}_{k}=\mathbf{H}_{k} \mathbf{x}_{k}+\mathbf{w}_{k}
$$

where $\mathbf{x}_{k}=\left[x_{k}\left[-\frac{N}{2}\right], x_{k}\left[-\frac{N}{2}+1\right], \ldots, x_{k}\left[\frac{N}{2}-1\right]\right]^{T} \cdot \mathbf{y}_{k}$ and $\mathbf{w}_{k}$ are defined in a similar way as $\mathbf{x}_{k} . \mathbf{w}_{k}$ is a white complex Gaussian noise vector of covariance matrix $\sigma^{2} \mathbf{I}_{N}$ and $\mathbf{H}_{k}$ is the $N \times N$ channel matrix. The elements of $\mathbf{H}_{k}$ can be written in terms of equivalent channel taps [19] $\left\{g_{l, k}\left(q T_{s}\right)=g_{l}(k T+\right.$ $\left.\left.q T_{s}\right)\right\}$

$$
\begin{aligned}
& {\left[\mathbf{H}_{k}\right]_{n, m}=} \\
& \quad \frac{1}{N} \sum_{l=0}^{L^{\prime}-1}\left[e^{-j 2 \pi\left(\frac{m}{N}-\frac{1}{2}\right) \cdot l} \sum_{q=0}^{N-1} g_{l, k}\left(q T_{s}\right) e^{j 2 \pi \frac{m-n+\nu}{N} q}\right]
\end{aligned}
$$

or in terms of physical channel parameters [15] (delays $\left\{\tau_{l}\right\}$ and CGs $\left.\left\{\alpha_{l, k}\left(q T_{s}\right)=\alpha_{l}\left(k T+q T_{s}\right)\right\}\right)$, yielding:

$$
\begin{aligned}
& {\left[\mathbf{H}_{k}\right]_{n, m}=} \\
& \quad \frac{1}{N} \sum_{l=0}^{L-1}\left[e^{-j 2 \pi\left(\frac{m}{N}-\frac{1}{2}\right) \tau_{l}} \sum_{q=0}^{N-1} \alpha_{l, k}\left(q T_{s}\right) e^{j 2 \pi \frac{m-n+\nu}{N} q}\right] .
\end{aligned}
$$

$L^{\prime}<N_{g}$ is the number of channel taps and $L$ the number of paths. The delays are normalized by $T_{s}$ and not necessarily integers $\left(\tau_{l}<N_{g}\right)$. The $L$ elements of $\left\{\alpha_{l, k}\left(q T_{s}\right)\right\}$ are uncorrelated. However, the $L^{\prime}$ elements of $\left\{g_{l, k}\left(q T_{s}\right)\right\}$ are correlated, unless the delays are multiple of $T_{s}$ as often assumed in the literature. These channel taps and CGs are wide-sense stationary (WSS), narrow-band zero-mean complex Gaussian processes of variances $\sigma_{g_{l}}{ }^{2}$ and ${\sigma_{\alpha_{l}}}^{2}$, respectively, and with the so-called Jakes' power spectrum of maximum Doppler frequency $f_{d}$ [20]. The average energy of the channel is normalized to one, i.e., $\sum_{l=0}^{L^{\prime}-1} \sigma_{g_{l}}{ }^{2}=1$ and $\sum_{l=0}^{L-1} \sigma_{\alpha_{l}}{ }^{2}=1$. Let us define the $N \times 1$ vector:

$$
\boldsymbol{\alpha}_{l, k}=\left[\alpha_{l, k}(0), \ldots, \alpha_{l, k}\left((N-1) T_{s}\right)\right]^{T}
$$

The correlation matrix of $\boldsymbol{\alpha}_{l, k}$ for the time-lag $p, \mathbf{R}_{\boldsymbol{\alpha}_{l}}^{(p)}=$ $\mathbb{E}\left[\boldsymbol{\alpha}_{l, k} \boldsymbol{\alpha}_{l, k-p}{ }^{H}\right]$, is given by:

$$
\left[\mathbf{R}_{\boldsymbol{\alpha}_{l}}^{(p)}\right]_{n, m}=\sigma_{\alpha_{l}}{ }^{2} J_{0}\left(2 \pi f_{d} T_{s}\left(n-m+p N_{b}\right)\right)
$$

In the next sections, we present the derivations for the second approach (physical channel). The results of the first approach (channel taps) can be easily deduced by replacing $L$ by $L^{\prime}$ and the set of delays $\left\{\tau_{l}\right\}$ by $\left\{l, l=0: L^{\prime}-1\right\}$.

\section{B. BEM Channel Model}

In each OFDM block, there are $N$ samples to be estimated for each path CG due to the fast time-variation of the channel, yielding a total of $L N$ samples for the whole channel and for each block. In order to reduce the number of parameters 
to be estimated, we resort to the Basis Expansion Model (BEM). In this section, our aim is to accurately model the time-variation of $\alpha_{l, k}\left(q T_{s}\right)$ from $q=0$ to $N-1$ by using a BEM. The purpose of using a BEM is to approximate $\boldsymbol{\alpha}_{l, k}$ as the weighted sum of just a few basis function $\mathbf{b}_{d}$, as follows:

$$
\boldsymbol{\alpha}_{l, k}=\boldsymbol{\alpha}_{\mathbf{B E M}_{l, k}}+\boldsymbol{\xi}_{l, k}=\mathbf{B} \cdot \mathbf{c}_{l, k}+\boldsymbol{\xi}_{l, k}
$$

where $\mathbf{B}=\left[\mathbf{b}_{0}, \ldots, \mathbf{b}_{N_{c}-1}\right]$ is a $N \times N_{c}$ matrix that collects the $N_{c}$ basis functions $\mathbf{b}_{d}$. Vector $\mathbf{c}_{l, k}=\left[c_{l, k}[0], \ldots, c_{l, k}\left[N_{c}-\right.\right.$ 1] $]^{T}$ represents the $N_{c}$ BEM coefficients for the $l$ th $\mathrm{CG}$ of the $k$ th OFDM symbol, and $\boldsymbol{\xi}_{l, k}$ represents the corresponding BEM modeling error, which is assumed to be minimized in the mean square error (MSE) sense [21]. Under this criterion, the optimal BEM coefficients and the corresponding model error are given by:

$$
\begin{aligned}
& \mathbf{c}_{l, k}=\left(\mathbf{B}^{H} \mathbf{B}\right)^{-1} \mathbf{B}^{H} \boldsymbol{\alpha}_{l, k} \\
& \boldsymbol{\xi}_{l, k}=\left(\mathbf{I}_{N}-\mathbf{S}\right) \boldsymbol{\alpha}_{l, k}
\end{aligned}
$$

where $\mathbf{S}=\mathbf{B}\left(\mathbf{B}^{H} \mathbf{B}\right)^{-1} \mathbf{B}^{H}$ is a $N \times N$ matrix.

Various traditional BEM designs have been reported to model the channel time-variations, e.g., the Complex Exponential BEM (CE-BEM), the Polynomial BEM (P-BEM) or the Karhuen-Loeve BEM (DKL-BEM) for instance [18][22].

From now on, we can describe the OFDM system model derived previously in terms of the BEM. Substituting (6) in (1) yields after some algebra:

$$
\mathbf{y}_{k}=\mathcal{K}_{k}(\nu) \cdot \mathbf{c}_{k}+\boldsymbol{\epsilon}_{k}+\mathbf{w}_{k}
$$

where the $L N_{c} \times 1$ vector $\mathbf{c}_{k}$ and the $N \times L N_{c}$ matrix $\mathcal{K}_{k}$ are given by:

$$
\begin{aligned}
\mathbf{c}_{k} & =\left[\mathbf{c}_{0, k}^{T}, \ldots, \mathbf{c}_{L-1, k}^{T}\right]^{T} \\
\mathcal{K}_{k}(\nu)= & \frac{1}{N}\left[\mathbf{Z}_{0, k}(\nu), \ldots, \mathbf{Z}_{L-1, k}(\nu)\right] \\
\mathbf{Z}_{l, k}(\nu)= & {\left[\mathbf{M}_{0}(\nu) \operatorname{diag}\left\{\mathbf{x}_{k}\right\} \mathbf{f}_{l}, \ldots,\right.} \\
& \left.\quad \mathbf{M}_{N_{c}-1}(\nu) \operatorname{diag}\left\{\mathbf{x}_{k}\right\} \mathbf{f}_{l}\right]
\end{aligned}
$$

where vector $\mathbf{f}_{l}$ is the $l$ th column of the $N \times L$ Fourier matrix $\mathbf{F}$ that depends on the delays distribution (defined by [14]), and $\mathbf{M}_{d}(\nu)$ is a $N \times N$ matrix given by:

$$
\begin{aligned}
{[\mathbf{F}]_{n, l} } & =e^{-j 2 \pi\left(\frac{n}{N}-\frac{1}{2}\right) \tau_{l}} \\
{\left[\mathbf{M}_{d}(\nu)\right]_{n, m} } & =\sum_{q=0}^{N-1}[\mathbf{B}]_{q, d} e^{j 2 \pi \frac{m-n+\nu}{N} q}
\end{aligned}
$$

The second component in (8), $\boldsymbol{\epsilon}_{k}$, represents the approximation error in the observation model.

\section{The AR Model for $\boldsymbol{c}_{k}$}

From (7), we get that the optimal BEM coefficients $\mathbf{c}_{l, k}$ are correlated complex Gaussian variables with zero-means and correlation matrix given by:

$$
\begin{aligned}
\mathbf{R}_{\mathbf{c}_{l}}^{(p)} & =\mathrm{E}\left[\mathbf{c}_{l, k} \mathbf{c}_{l, k-p}^{H}\right] \\
& =\left(\mathbf{B}^{H} \mathbf{B}\right)^{-1} \mathbf{B}^{H} \mathbf{R}_{\boldsymbol{\alpha}_{l}}^{(p)} \mathbf{B}\left(\mathbf{B}^{H} \mathbf{B}\right)^{-1}
\end{aligned}
$$

Since the coefficients $\mathbf{c}_{l, k}$ are correlated Gaussian variables, their dynamics can be well approached by an auto-regressive
(AR) process [23] [15] . A complex AR process of order $I$, denoted $\tilde{\mathbf{c}}_{l, k}$, can be generated as:

$$
\tilde{\mathbf{c}}_{l, k}=\sum_{i=1}^{I} \mathbf{A}^{(i)} \tilde{\mathbf{c}}_{l, k-i}+\mathbf{u}_{l, k}
$$

where $\mathbf{A}^{(1)}, \ldots, \mathbf{A}^{(I)}$ are $N_{c} \times N_{c}$ matrices and $\mathbf{u}_{l, k}$ is a $N_{c} \times 1$ complex Gaussian vector with covariance matrix $\mathbf{U}_{l}$. From [15], it is sufficient to choose $I=1$ to correctly capture the coefficients dynamics. The matrices $\mathbf{A}^{(1)}=\mathbf{A}$ and $\mathbf{U}_{l}$ are the AR model parameters. The standard choice [23] to compute those parameters is to impose that the correlation matrices $\mathbf{R}_{\tilde{\mathbf{c}}_{l}}^{(p)}$ of the approximate process perfectly match the correlation matrices $\mathbf{R}_{\mathbf{c}_{l}}^{(p)}$ of the true process for lag $p \in\{-1,0,1\}$ in (13). Imposing this correlation matching constraint, the parameters of the AR process can be computed by the set of the YuleWalker equations defined as:

$$
\begin{aligned}
\mathbf{A} & =\mathbf{R}_{\mathbf{c}_{l}}^{(1)}\left(\mathbf{R}_{\mathbf{c}_{l}}^{(0)}\right)^{-1} \\
\mathbf{U}_{l} & =\mathbf{R}_{\mathbf{c}_{l}}^{(0)}-\mathbf{A} \mathbf{R}_{\mathbf{c}_{l}}^{(-1)}
\end{aligned}
$$

Using (14), we obtain the AR model of order 1 for $\tilde{\mathbf{c}}_{k}$ :

$$
\tilde{\mathbf{c}}_{k}=\mathcal{A} \cdot \tilde{\mathbf{c}}_{k-1}+\mathbf{u}_{k}
$$

where $\mathcal{A}=\operatorname{blkdiag}\{\mathbf{A}, \ldots, \mathbf{A}\}$ is a $L N_{c} \times L N_{c}$ matrix and $\mathbf{u}_{k}=\left[\mathbf{u}_{0, k}^{T}, \ldots, \mathbf{u}_{L-1, k}^{T}\right]^{T}$ is a $L N_{c} \times 1$ zeromean complex Gaussian vector with covariance matrix $\mathbf{U}=$ blkdiag $\left\{\mathbf{U}_{0}, \ldots, \mathbf{U}_{L-1}\right\}$.

\section{EM ALGORITHM}

First, we consider a block of $K$ OFDM received symbols. Let $\mathbf{y}=\left[\mathbf{y}_{0}^{T}, \ldots, \mathbf{y}_{K-1}^{T}\right]^{T}$ and $\mathbf{c}=\left[\mathbf{c}_{0}^{T}, \ldots, \mathbf{c}_{K-1}\right]^{T}$. The objective is to jointly estimate the CFO $\nu$ and the BEM coefficients $\mathbf{c}$ of the path CGs based on the set of the $K$ OFDM received signals. It is noteworthy that if $\nu$ is known, the BEM coefficients can be estimated by using a Kalman filter [15]. In our specific context, both $\nu$ and $\mathbf{c}$ are unknown. Therefore we propose to use the EM algorithm, which has been extensively explained in [24]. The EM algorithm is an iterative method to find the ML estimates of parameters in the presence of unobserved data. The algorithm comprises two steps : the expectation step (E-step) and the maximization step (M-step). We describe an EM algorithm for our model.

We suppose that the transmitted symbols $\mathbf{x}_{k}$ are known (this configuration is called Data Aided (DA), and corresponds to the acquisition task). We consider the received data $\mathbf{y}$ as incomplete data, and define the complete data as $\mathbf{z} \stackrel{\text { def }}{=}\left[\mathbf{y}^{T}, \mathbf{c}^{T}\right]^{T}$. Since the state is described by first order Markov model, the likelihood function of the complete data is given by:

$$
p(\mathbf{z} ; \nu)=p\left(\mathbf{c}_{0}\right) \prod_{k=1}^{K-1} p\left(\mathbf{c}_{k} \mid \mathbf{c}_{k-1}\right) \prod_{k=0}^{K-1} p\left(\mathbf{y}_{k} \mid \mathbf{c}_{k} ; \nu\right)
$$

Then, from (8) (the error term $\epsilon_{k}$ is neglected), we compute 
the log-likelihood of the complete data:

$$
\begin{array}{r}
\ln (p(\mathbf{z} ; \nu))=C-\frac{1}{\sigma^{2}} \sum_{k=0}^{K-1}\left(\mathbf{y}_{k}-\mathbf{m}_{k}(\nu)\right)^{H}\left(\mathbf{y}_{k}-\mathbf{m}_{k}(\nu)\right) \\
+\ln p\left(\mathbf{c}_{0}\right)+\sum_{k=1}^{K-1} \ln p\left(\mathbf{c}_{k} \mid \mathbf{c}_{k-1}\right)
\end{array}
$$

where $C$ is a constant and $\mathbf{m}_{k}(\nu)=\mathcal{K}_{k}(\nu) \mathbf{c}_{k}$ is the mean vector. Each iteration $i=0,1, \ldots$ of the EM algorithm for estimating $\nu$ from $\mathbf{y}$ consists in two steps:

E-step : given the measurements $\mathbf{y}$ and the latest estimate $\hat{\nu}^{(i)}$ from the previous iteration, we calculate:

$$
Q\left(\nu, \hat{\nu}^{(i)}\right) \stackrel{\text { def }}{=} \mathbb{E}_{\mathbf{c} \mid \mathbf{y}, \hat{\nu}^{(i)}}[\ln p(\mathbf{z} ; \nu)]
$$

M-step : this step finds $\hat{\nu}^{(i+1)}$, the value of $\nu$ which maximizes $Q\left(\nu, \hat{\nu}^{(i)}\right)$ over all possible values of $\nu$ :

$$
\hat{\nu}^{(i+1)}=\operatorname{argmax}_{\nu} Q\left(\nu, \hat{\nu}^{(i)}\right)
$$

This procedure is repeated until the sequence $\hat{\nu}^{(0)}, \hat{\nu}^{(1)}, \ldots$ converges.

Due to the Gauss-Markov nature of the problem, the computation of the $Q$ function can be expressed as (see Appendix A):

$$
\begin{array}{r}
Q\left(\nu, \hat{\nu}^{(i)}\right)=-\frac{1}{\sigma^{2}} \sum_{k=0}^{K-1} \operatorname{Tr}\left\{\mathcal{K}_{k}(\nu) \mathbf{S}_{k \mid K}^{(i)} \mathcal{K}_{k}^{H}(\nu)\right. \\
\left.\left(\mathbf{y}_{k}-\mathcal{K}_{k}(\nu) \hat{\mathbf{c}}_{k \mid K}^{(i)}\right)\left(\mathbf{y}_{k}-\mathcal{K}_{k}(\nu) \hat{\mathbf{c}}_{k \mid K}^{(i)}\right)^{H}\right\}
\end{array}
$$

where $\quad \hat{\mathbf{c}}_{k \mid K}^{(i)} \stackrel{\text { def }}{=} \quad \mathbb{E}_{\mathbf{c} \mid \mathbf{y}, \hat{\nu}^{(i)}}\left[\mathbf{c}_{k}\right] \quad$ and $\quad \mathbf{S}_{k \mid K}^{(i)} \quad \stackrel{\text { def }}{=}$ $\mathbb{E}_{\mathbf{c} \mid \mathbf{y}, \hat{\nu}}(i)\left[\left(\mathbf{c}_{k}-\hat{\mathbf{c}}_{k \mid K}^{(i)}\right)\left(\mathbf{c}_{k}-\hat{\mathbf{c}}_{k \mid K}^{(i)}\right)^{H}\right]$. The required terms $\hat{\mathbf{c}}_{k \mid K}^{(i)}$ and $\mathbf{S}_{k \mid K}^{(i)}$ can actually be computed for all $k=0, \ldots, K-1$ from the fixed interval Kalman smoother (using the parameter estimate at iteration $i$ ), since our model ( 8 in neglecting the error term $\epsilon_{k}$ ) becomes a so-called Gaussian linear model for the estimation of $c_{k}$ assuming previous knowledge of $\nu$. The E-step is thus an Estimation-step for the BEM coefficients vector. The smoother consists of a backward pass that follows the standard Kalman filter Forward recursion given by:

Forward recursion:

Time Update Equations (TUE):

$$
\begin{aligned}
\hat{\mathbf{c}}_{k \mid k-1}^{(i)} & =\mathcal{A} \hat{\mathbf{c}}_{k-1 \mid k-1}^{(i)} \\
\mathbf{S}_{k \mid k-1}^{(i)} & =\mathcal{A} \mathbf{S}_{k-1 \mid k-1}^{(i)} \mathcal{A}^{H}+\mathbf{U}
\end{aligned}
$$

Measurement Update Equations (MUE):

\section{Backward recursion:}

$$
\begin{aligned}
\mathbf{J}_{k} & =\mathbf{S}_{k-1 \mid k-1}^{(i)} \mathcal{A}^{H} \mathbf{S}_{k \mid k-1}^{(i)} \\
\hat{\mathbf{c}}_{k-1 \mid K}^{(i)} & =\hat{\mathbf{c}}_{k-1 \mid k-1}^{(i)}+\mathbf{J}_{k}\left(\hat{\mathbf{c}}_{k \mid K}^{(i)}-\hat{\mathbf{c}}_{k \mid k-1}^{(i)}\right) \\
\mathbf{S}_{k-1 \mid K}^{(i)} & =\mathbf{S}_{k-1 \mid k-1}^{(i)}+\mathbf{J}_{k}\left(\mathbf{S}_{k \mid K}^{(i)}-\mathbf{S}_{k \mid k-1}^{(i)}\right) \mathbf{J}_{k}^{H}
\end{aligned}
$$

It is noteworthy that the EM is a computationally less demanding technique than MAP [25], since it does not require batch processing and the matrices to be inverted are only of size $L N_{c} \times L N_{c}$. The BEM-based MAP technique requires batch processing and the inversion of $\left(K L N_{c} \times K L N_{c}\right)$ matrices. We show in Section $\mathrm{V}$ that the MSE performances of the two methods are very close to each other.

\section{HYBRID CRAMÉR-RAO BOUNDS (HCRB)}

\section{A. Introduction}

The CRB for the fast time-varying path CGs has been first computed in [17] for the OFDM context. However, the calculated $\mathrm{CRB}$ assumed a BEM model for the channels. Hence, the CRB of the CGs derived in [17] cannot be considered as the true CRB but as a BEM-based CRB since it is based on the CRB of the BEM coefficients. The exact CRB for the CGs is the one that does not assume a BEM.

In this Section, we propose to derive the true $\mathrm{CRB}$ for the estimation of the CGs together with the CFO, in the presence of very high mobility. The CFO being deterministic, this yields to calculate a Hybrid CRB. The CRBs provide a lower bound on the Mean Square Error (MSE) achievable by any unbiased estimator. We give the general expression of the Hybrid Cramér-Rao Bound (HCRB). The HCRB is particularly suited for problems where the parameters to be estimated encompass both deterministic (here $\nu$ ) and random (here $\boldsymbol{\alpha}$ ) components, with availability of the a priori information $(p(\boldsymbol{\alpha}))$. An off-line scenario is considered, i.e. the receiver waits until the whole observation frame of $K$ OFDM symbols $\mathbf{y}=\left[\mathbf{y}_{0}^{T}, \ldots, \mathbf{y}_{K-1}^{T}\right]^{T}$ has been received to perform parameter estimation.

Vector $\boldsymbol{\alpha}$ is defined as follows:

$$
\begin{aligned}
\boldsymbol{\alpha} & =\left[\boldsymbol{\alpha}_{0}^{T}, \ldots, \boldsymbol{\alpha}_{K-1}^{T}\right]^{T} \\
\boldsymbol{\alpha}_{k} & =\left[\boldsymbol{\alpha}_{0, k}^{T}, \ldots, \boldsymbol{\alpha}_{L-1, k}^{T}\right]^{T}
\end{aligned}
$$

where the vector $\boldsymbol{\alpha}_{l, k}$ is defined in (4).

Let $\boldsymbol{\mu}=\left[\boldsymbol{\alpha}^{T}, \nu\right]^{T}$ be the hybrid vector to be estimated. The HCRB has been initially proposed in [26] as:

$$
\mathbb{E}_{\mathbf{y}, \boldsymbol{\alpha} \mid \nu}\left[\left.(\hat{\boldsymbol{\mu}}(\mathbf{y})-\boldsymbol{\mu})(\hat{\boldsymbol{\mu}}(\mathbf{y})-\boldsymbol{\mu})^{H}\right|_{\nu}\right] \geq \mathbf{H C R B}
$$

where $\mathbf{X} \geq \mathbf{Y}$ is interpreted as meaning that the matrix $\mathbf{X}-\mathbf{Y}$ is positive semi-definite. We partition the HCRB matrix as follows:

$$
\text { HCRB }=\left[\begin{array}{cc}
\mathbf{H C R B}_{\boldsymbol{\alpha}} & \mathrm{HCRB}_{12} \\
\mathbf{H C R B}_{21} & \mathrm{HCRB}_{\nu}
\end{array}\right]
$$

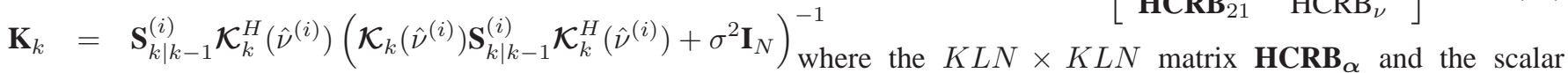
$\hat{\mathbf{c}}_{k \mid k}^{(i)}=\hat{\mathbf{c}}_{k \mid k-1}^{(i)}+\mathcal{K}_{k}\left(\hat{\nu}^{(i)}\right)\left(\mathbf{y}_{k}-\mathcal{K}_{k}\left(\hat{\nu}^{(i)}\right) \hat{\mathbf{c}}_{k \mid k-1}^{(i)}\right)$

$\mathbf{S}_{k \mid k}^{(i)}=\mathbf{S}_{k \mid k-1}^{(i)}-\mathbf{K}_{k} \mathcal{K}_{k}\left(\hat{\nu}^{(i)}\right) \mathbf{S}_{k \mid k-1}^{(i)}$ $\left({ }^{2} 3 \mathrm{FRB}_{\nu}\right.$ are the minimal bounds on the MSE of $\boldsymbol{\alpha}$ and $\nu$, respectively, and the vectors $\mathbf{H C R B} \mathbf{B}_{12}$ and $\mathbf{H C R B} \mathbf{B}_{21}$ are the cross-terms. Note that for notational convenience, we drop the 
dependence of these submatrices on $\nu$. The $\mathrm{HCRB}^{1}$ is the inverse of the Hybrid Information Matrix (HIM), which can be written as:

$$
\mathbf{H i}(\nu)=\mathbb{E}_{\boldsymbol{\alpha}}[\mathbf{F i}(\boldsymbol{\alpha}, \nu)]+\mathbb{E}_{\boldsymbol{\alpha} \mid \nu}\left[-\left.\Delta_{\boldsymbol{\mu}}^{\boldsymbol{\mu}} \ln (p(\boldsymbol{\alpha} \mid \nu))\right|_{\nu}\right]
$$

where $p(\boldsymbol{\alpha} \mid \nu)$ is the prior distribution and $\mathbf{F i}(\boldsymbol{\alpha}, \nu)$ is the Fisher Information Matrix (FIM) defined as:

$$
\boldsymbol{F i}(\boldsymbol{\alpha}, \nu)=\mathbb{E}_{\mathbf{y} \mid \boldsymbol{\alpha}, \nu}\left[-\left.\Delta_{\boldsymbol{\mu}}^{\boldsymbol{\mu}} \ln (p(\mathbf{y} \mid \boldsymbol{\alpha}, \nu))\right|_{\nu}\right]
$$

where $p(\mathbf{y} \mid \boldsymbol{\alpha}, \nu)$ is the conditional probability density function of $\mathbf{y}$ given $\boldsymbol{\alpha}$ and $\nu$. Since the deterministic and the random parts of the hybrid vector are statistically independent, i.e., $p(\boldsymbol{\alpha} \mid \nu)=p(\boldsymbol{\alpha})$, then after some algebraic manipulations, the second component of the HIM can be written as [27]:

$$
\begin{aligned}
\mathbb{E}_{\boldsymbol{\alpha} \mid \nu}\left[-\Delta_{\boldsymbol{\mu}}^{\boldsymbol{\mu}}\right. & \left.\left.\ln (p(\boldsymbol{\alpha} \mid \nu))\right|_{\nu}\right]= \\
& {\left[\begin{array}{cc}
\mathbb{E}_{\boldsymbol{\alpha}}\left[-\Delta_{\boldsymbol{\alpha}}^{\boldsymbol{\alpha}} \ln (p(\boldsymbol{\alpha}))\right] & \mathbf{0}_{K L N, 1} \\
\mathbf{0}_{1, K L N} & 0
\end{array}\right] }
\end{aligned}
$$

In the DA context, the transmitted data symbols $\mathbf{x}$ are known at the receiver and then no averaging over the data is required. Hence, the probability density function depends on $\mathbf{x}$, and so do the bound.

Finally, the mean MSE of the estimation of $\boldsymbol{\alpha}$ is lowerbounded by:

$$
\overline{\mathrm{HCRB}}_{\boldsymbol{\alpha}}=\frac{1}{K L N} \operatorname{Tr}\{\mathbf{H C R B} \boldsymbol{\alpha}\}
$$

The HCRB associated with the estimation of $\nu, \mathrm{HCRB}_{\nu}$, is given by (27).

\section{B. Expression of the $H C R B$}

The closed-form exact expression for the HCRB related to the estimations of $\boldsymbol{\alpha}$ and $\nu$ in the Data Aided (DA) mode of OFDM systems is presented hereafter. The details of the derivation are given in Appendix B, yielding:

$$
\begin{aligned}
\text { HCRB }=\left(\operatorname { b l k d i a g } \left\{\mathcal{J}_{0}(\nu), \ldots, \mathcal{J}_{K-1}(\nu),\right.\right. \\
\left.\left.\mathbb{E}_{\boldsymbol{\alpha}}\left[\operatorname{Fi}_{22}(\boldsymbol{\alpha}, \nu)\right]\right\}+\operatorname{blkdiag}\left\{\mathbf{R}_{\boldsymbol{\alpha}}^{-1}, 0\right\}\right)^{-1}
\end{aligned}
$$

where $\mathcal{J}_{k}(\nu)$ is given by (42), $\mathbb{E}_{\boldsymbol{\alpha}}\left[\mathrm{Fi}_{22}(\boldsymbol{\alpha}, \nu)\right]$ is given by (44) and $\mathbf{R}_{\boldsymbol{\alpha}}$ is given by (45).

\section{Simulation Results}

For all our simulations the channel autocorrelation function is assumed to be given by the widely accepted Jakes' model, as stated in Section II.

System parameters in our simulations follow the WiMAX mobile standard [28], with $N=128$ and $1 / T_{s}=1.25 \mathrm{MHz}$, yielding a subcarrier spacing of $10.94 \mathrm{KHz}$ which is the value imposed by WiMAX (section 2.3 WIMAX Physical layer in [28]). The carrier frequency is set to $3.5 \mathrm{GHz}$.

The Rayleigh channel model is the channel A from [28] ( $L=6$ paths and maximum delay $\tau_{\max }=3.1 T_{s}$, see Table I). The data symbols are drawn from normalized 4QAM constellations. We set $N_{g}=\frac{N}{8}$. Three different normalized

${ }^{1}$ We recall that, for a deterministic parameter, Standard Cramér-Rao Bound (SCRB) would be directly the inverse of the Fisher Information Matrix (FIM).

\begin{tabular}{|c|c|c|}
\hline Path number & Relative Power (dB) & Delay (nsec) \\
\hline \hline 0 & 0 & 0 \\
\hline 1 & -1 & 310 \\
\hline 2 & -9 & 710 \\
\hline 3 & -10 & 1,090 \\
\hline 4 & -15 & 1,730 \\
\hline 5 & -20 & 2,510 \\
\hline
\end{tabular}

TABLE I

ITU MULTIPATH CHANNEL MODEL - CHANNEL A [28]

Doppler frequencies have been selected, $f_{d} T=0.05, f_{d} T=$ 0.1 and $f_{d} T=0.15$, corresponding to a mobile terminal moving at speeds of $130 \mathrm{Km} / \mathrm{h}, 270 \mathrm{Km} / \mathrm{h}$ and $400 \mathrm{Km} / \mathrm{h}$, respectively. The BEM model used for the simulation is the polynomial BEM. The normalized CFO $\nu$ has been set to 0.35 , corresponding to an oscillator tolerance of $1 \mathrm{ppm}$. The mean MSE of the estimations of $\boldsymbol{\alpha}$ and the MSE of the estimation of $\nu$ are defined as follows:

$$
\begin{array}{lll}
\overline{\operatorname{MSE}}_{\alpha} & \stackrel{\text { def }}{=} \frac{1}{K N L} \mathbb{E}\left[(\hat{\boldsymbol{\alpha}}-\boldsymbol{\alpha})^{H}(\hat{\boldsymbol{\alpha}}-\boldsymbol{\alpha})\right] \\
\mathrm{MSE}_{\nu} & \stackrel{\text { def }}{=} \mathbb{E}\left[|\hat{\nu}-\nu|^{2}\right]
\end{array}
$$

where the expectation is estimated via monte-carlo simulations in the following.

\section{A. Acquisition algorithm}

First, the impact of the number of EM iterations on CFO estimation has been investigated for different speeds. Fig. 1 shows the mean over 100 realizations of the $\mathrm{CFO}$ recursive estimate $\left(\hat{\nu}^{(i)}\right)$ versus the number of EM iterations $i$ for $E_{b} / N_{0}=20 \mathrm{~dB}$ and $K=2$. The normalized CFO $\nu$ has been set to 0.35 , corresponding to an oscillator tolerance of $1 \mathrm{ppm}$. This figure shows that the estimated CFO converges to the true value faster when the Doppler is lower. For $f_{d} T=0.05$, $f_{d} T=0.1, f_{d} T=0.15$, the algorithm converges after about $30,50,100$ iterations respectively. Hence, the convergence time strongly depends on the mobile speed. Fig. 2 illustrates this convergence with the $Q$-function as a function of $\nu$ for different values of iteration number $i$ and for $f_{d} T=0.1$ (always for a true value $\nu=0.35$ ). It is worthwhile to observe that the function does not present local maximas.

Next, the acquisition range for the $\mathrm{CFO}$ has been investigated for the most critical case $f_{d} T=0.15$. Fig. 3 shows the CFO estimate curves $\left(\hat{\nu}^{(i)}\right)$ versus the number of EM iterations for different values of $\nu$ selected in the range $[-1.2,1.3]$ with an increment of 0.1 (each curve corresponding to a given $\hat{\nu}$ is averaged over 100 realizations). It is shown that the algorithm is able to acquire the normalized CFO $\nu$ in the range $[-1.1,1.2]$, corresponding to about $\pm 3.4 \mathrm{ppm}$.

Fig. 4 shows the HCRB for the CG estimation (28) and CFO estimation (27) versus the block size $K$ for different values of $E_{b} / N_{0}$, again with $f_{d} T=0.1$ and $N_{c}=3 . K$ varies from 2 to 50 . As expected, it is observed that both bounds rapidly decrease when $K$ varies from 2 to 10 , and then decrease with a smoother slope. Hence, the gain in performance is important only for small values of $K$, then the gain becomes negligible. A good trade off would be to choose $K=10$, but the value of 


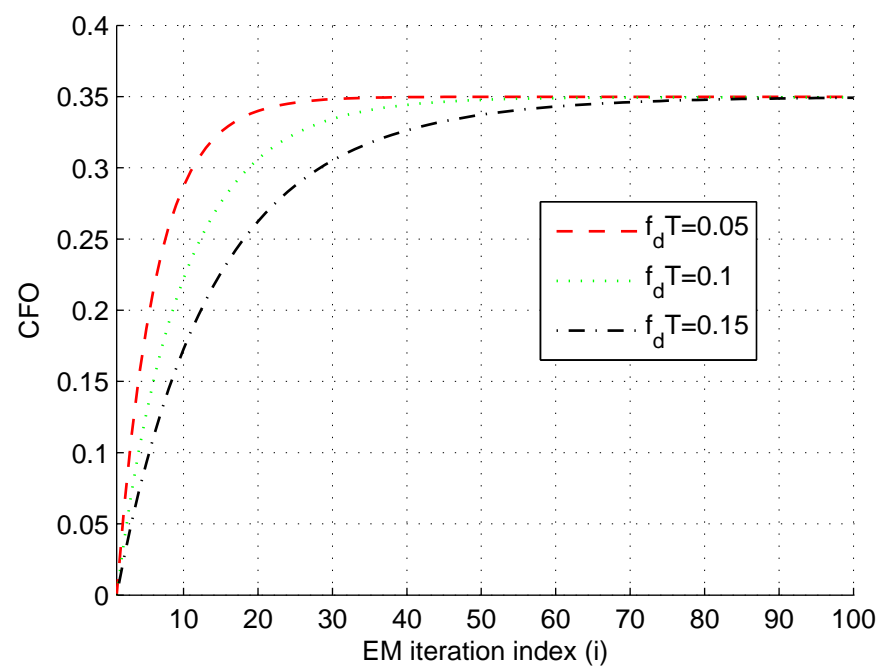

Fig. 1. An EM trajectory for different values of $f_{d} T$ with $E_{b} / N_{0}=20 \mathrm{~dB}$ and $K=2, \nu=0.35$

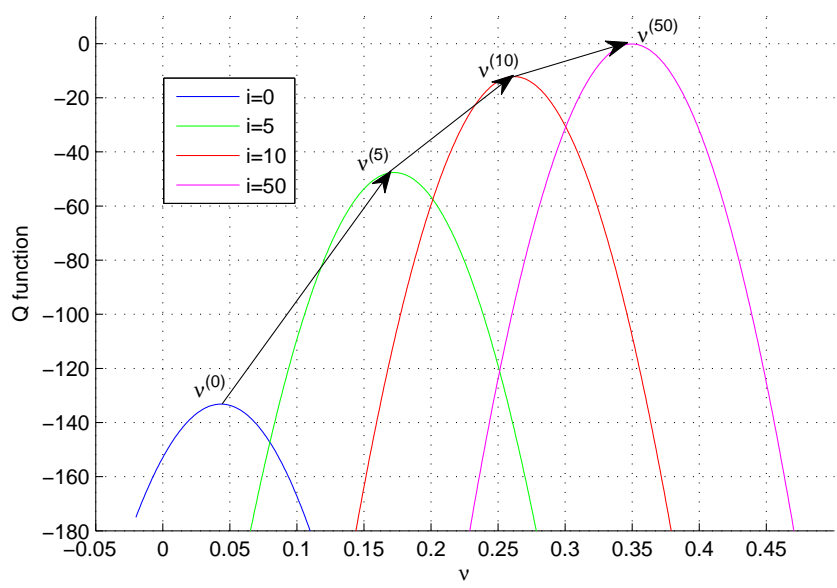

Fig. 2. $Q$ functions at $E_{b} / N_{0}=20 \mathrm{~dB}$ and $f_{d} T=0.1, K=2, \nu=0.35$

$K$ depends on the standard. A typical value is $K=2$. In the sequel, we will perform simulations with $K=2$ and $K=5$.

Fig. 5 shows the MSE performance of the path CG estimation for the forward step and the backward step. For reference, the HCRB has been plotted. The parameters values are $\nu=0.35, N_{c}=3$ and $f_{d} T=0.1$. Simulations have been performed for both $K=2$ and $K=5$. As expected the backward step consistently outperforms the performance of the forward step. This is understood since the backward step uses the whole received sequence. Note also that the backward step reaches the HCRB. On the other hand, the improvement given by the backward step is more important with $K=5$ than with $K=2$. The gain in performance as a function of the block size can also be observed with the HCRB in Fig. 4. The MSE for the MAP algorithm [25] has also been plotted. It is similar to that obtained with the proposed EM algorithm.

Fig. 6 shows the MSE performance of the CFO estimation for the proposed EM algorithm and the MAP algorithm. The MSE of CFO MAP estimate is far from the bound, and is

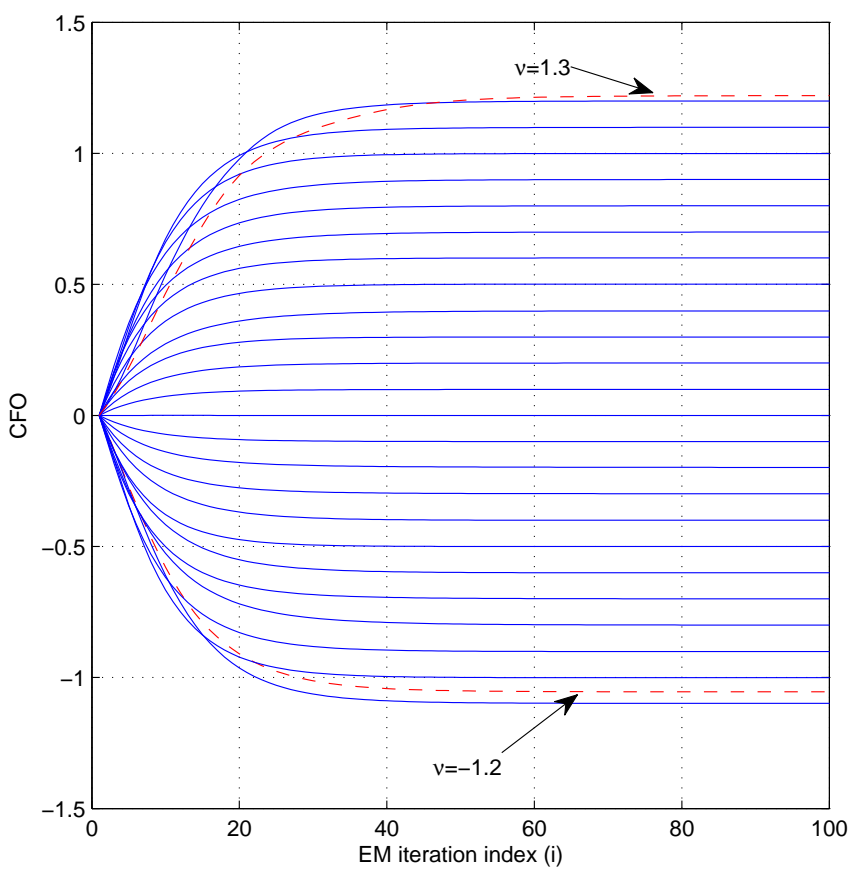

Fig. 3. EM trajectories for different values of $\nu$ at $E_{b} / N_{0}=20 \mathrm{~dB}$ and $f_{d} T=0.15, K=2$
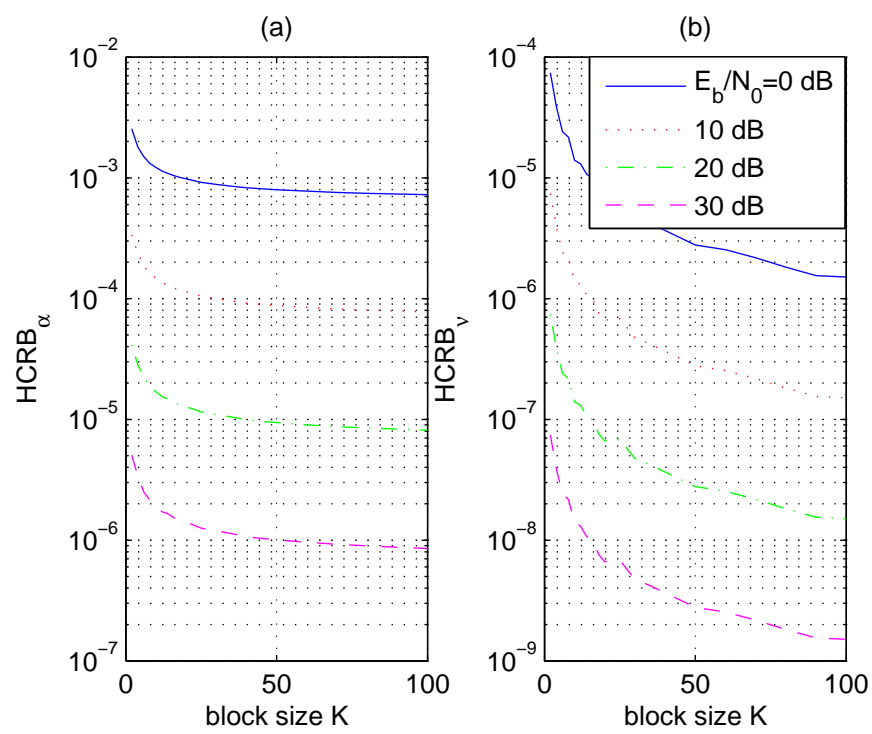

Fig. 4. HCRB versus $K$ for (a) the CG estimation, (b) the CFO estimation with $f_{d} T=0.1$ and $N_{c}=3$

quasi the same as the MSE of CFO EM estimate. Since we take the expectation of the hybrid FIM with respect to the channel statistics, the HCRB is not necessarily tight for the CFO estimation. This is similar to what is observed with the modified CRB for the CFO in the case of non-data aided systems where the expectation of the FIM is taken with respect to the unknown data before taking the inverse. 

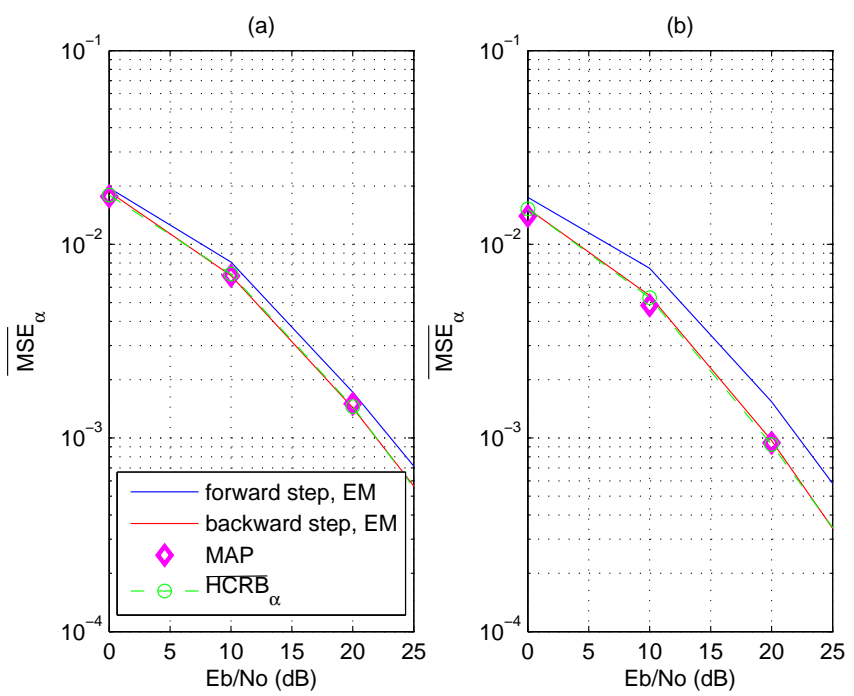

Fig. 5. MSE performance of the CGs estimation versus $E_{b} / N_{0}$ for (a) $K=2$, (b) $K=5 ; f_{d} T=0.1, N_{c}=3$
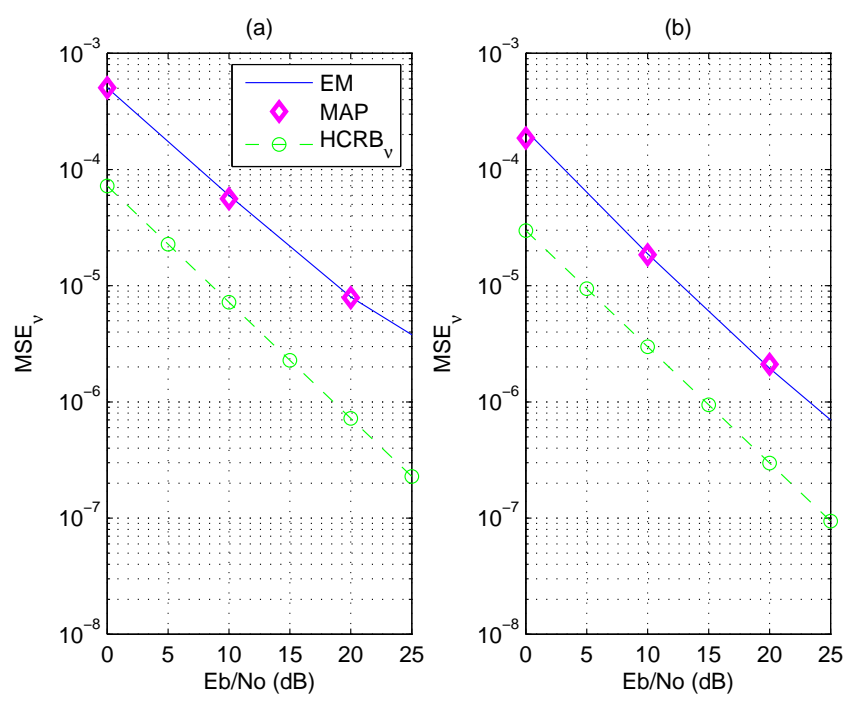

Fig. 6. MSE performance of the CFO estimation versus $E_{b} / N_{0}$ for (a) $K=2$, (b) $K=5 ; f_{d} T=0.1, N_{c}=3$

\section{B. Tracking algorithm}

The proposed EM algorithm is used as an acquisition algorithm on the first $K$ OFDM symbols of the frame, assuming the knowledge of all the transmitted symbols $\mathbf{x}_{k}, k=$ $0 \ldots K-1$. In order to propose a complete algorithm able to perform detection, we propose using the tracking algorithm of [15]. This tracking algorithm proceeds in order to jointly make $\mathrm{CG}$ estimation and data detection, based on pilots symbols evenly inserted in each OFDM symbol. The pilot positions are given by the set of pilot indices $\mathcal{P}=\left\{n L_{f}, n=0 \ldots N_{p}-1\right\}$, where $L_{f}$ is the distance between two adjacent pilots and $N_{p}$ is the number of pilots. The algorithm is an on-line algorithm, i.e. it operates on each OFDM symbol. The CG tracking is based on Kalman filter, using the CG estimated in the acquisition phase as an initial
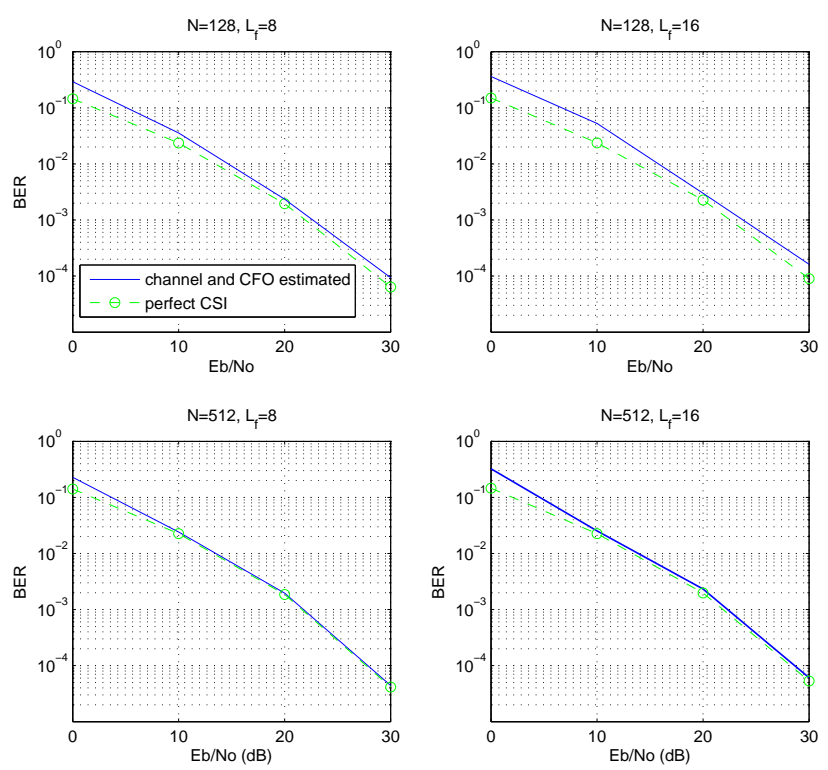

Fig. 7. BER of the proposed tracking algorithm for $L_{f}=8, L_{f}=16$ and $N=128, N=512$

value. It should be noted that we use the estimated CFO to remove its effect on the received symbols. The switch between our acquisition algorithm and this tracking algorithm makes sense since both algorithms rely on an AR(1) dynamical model of the BEM representation of the CG variations, used for the Kalman filter.

Fig. 7 shows the obtained BER for $N=128$ and $N=512$. For the sake of comparison, the performance of the algorithm obtained with perfect channel state information (CSI) is also given. As expected, the BER is closer to the one obtained with perfect CSI when using a higher number of pilots. Also, the BER with $N=512$ is more favorable than with $N=128$ (note that the bandwidth is larger with $N=512$, the subcarrier spacing remaining constant). Hence, to test the robustness of the algorithm in the sequel, we will choose the most unfavorable case $N=128$.

\section{Robustness of the tracking algorithm to imperfect delay and Doppler frequency knowledge}

Fig. 8 measures the effect of an imperfect delay knowledge on the BER performance of our proposed tracking algorithm. $\mathrm{SD}$ denotes the standard deviation of the time delay errors (modeled as zero mean Gaussian variables). As expected (common drawback to any parametric channel estimator), the algorithm performance decreases with respect to the delay error. However, the algorithm is not very sensitive to a delay error $S D<0.2 T_{s}$ in low SNR, and $S D<0.1 T_{s}$ in high SNR, for both $N_{p}=8$ and $N_{p}=16$. And when increasing the number of pilot subcarriers to $N_{p}=16$, sensibility to delay error decreases. These required performance for the delays acquisition can well be obtained when using high resolution algorithms [14], thanks to the quasi invariance of the delays (with respect to the scale of the sampling time $T_{s}$ ) during a 

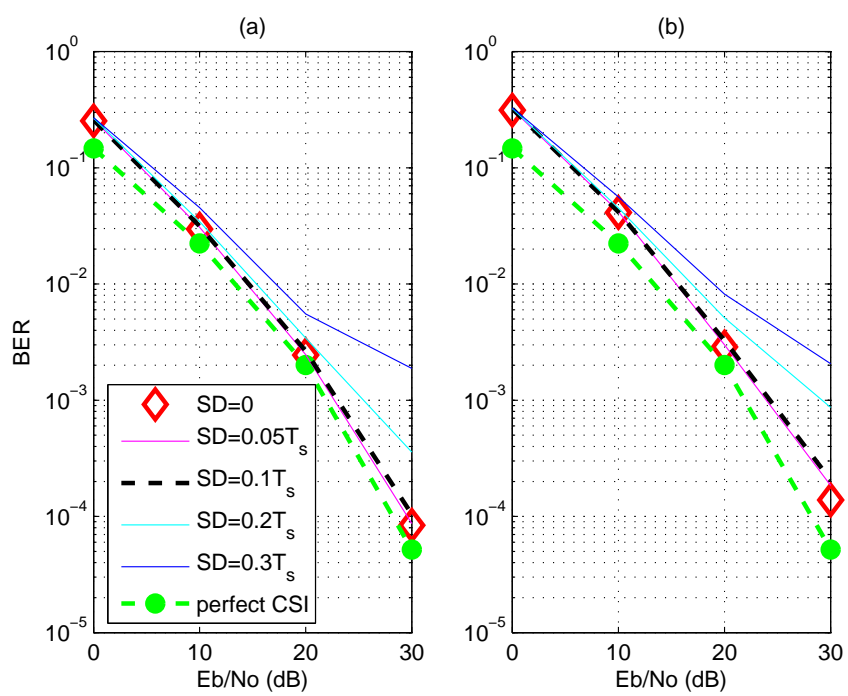

Fig. 8. BER of the proposed tracking algorithm for imperfect knowledge of the delays for (a) $N_{p}=16$, (b) $N_{p}=8$
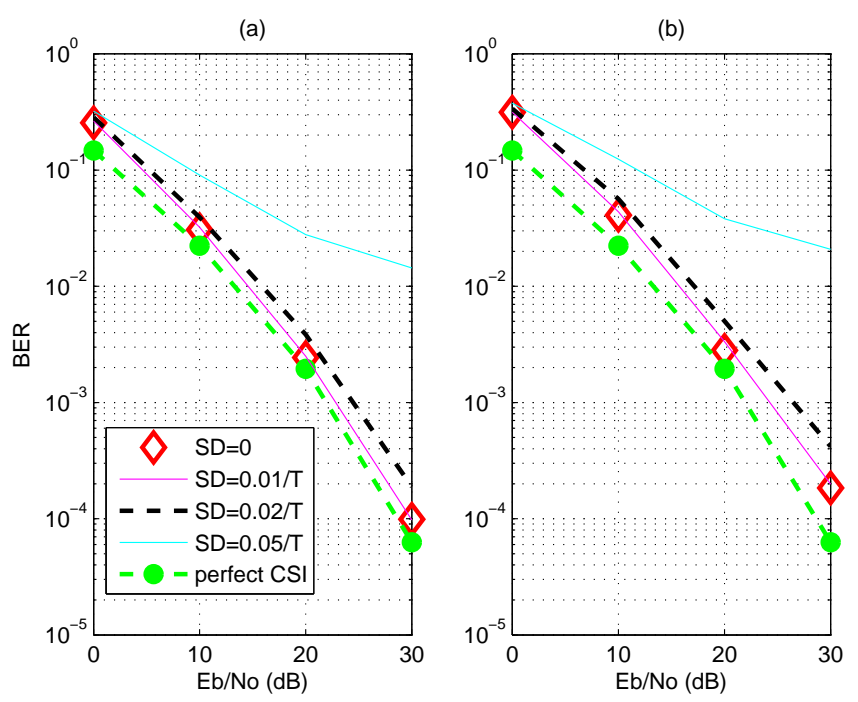

Fig. 9. BER of the proposed tracking algorithm for imperfect knowledge of the Doppler frequency for (a) $N_{p}=16$, (b) $N_{p}=8$

large number of OFDM symbols (see also performance in [15] obtained by the ESPRIT method [14]).

Robustness to imperfect Doppler frequency is investigated in Fig. 9. The algorithm is not sensitive to a Doppler frequency error $S D<0.01 / T$ for both $N_{p}=8$ and $N_{p}=16$. Similarly to the delay error, increasing the number of pilots increases the robustness to Doppler frequency errors.

We now study the effect of the wrong estimate of $L=6$ on our algorithm with $N_{p}=16$. A study on this issue was made in [15] for time-varying channels estimation (without CFO). $L e$ stands for the estimate of $L$. In case of an overestimated ( $L e=7$ and $L e=8$ ), it is shown that such a mismatch ends up in slight degrades of performance as shown in 10. However, in case of underestimated ( $L e=5$ and $L e=4)$, the performance of our algorithm suffers from this disappearing of paths especially at moderate and high $E_{b} / N_{0}$ region. This

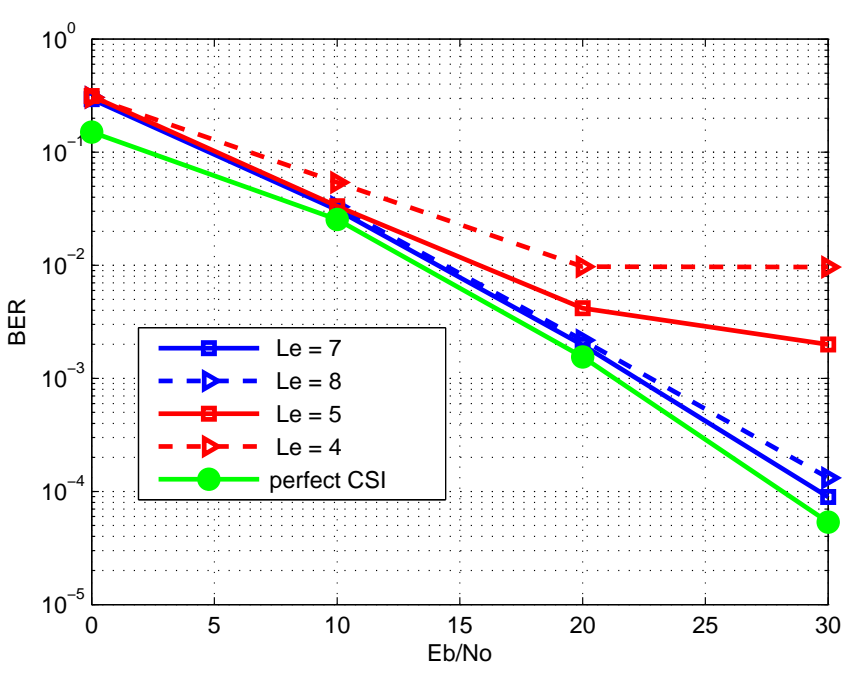

Fig. 10. BER of the proposed tracking algorithm for imperfect knowledge of the number of paths $L$

result is similar to the result obtained in [15]. However as [15], by using a robust method like the minimum description length (MDL) to estimate $L$, we can make our algorithm correctly performs without a priori knowledge of $L$.

\section{Performance comparison}

In this section, we compare our proposed algorithm with the algorithm of [9]. This algorithm is based on the extended Kalman filtering to carry out channel taps and CFO estimation together with data detection. Note that it operates on equivalent discrete channel model only, and not on the parametric channel model. The simulations presented in [9] have been carried out in Decision-Directed (DD) mode only (after the acquisition step), i.e. only decoded data symbols are used to perform the filtering. However, when introducing their algorithm, the authors also stated that in case of high mobility, pilot signals are also needed [9][29]. So to compare both algorithms, we insert pilots in the algorithm following the pilot scheme of our tracking algorithm [15]. We use the same parameters as in [9] to perform the simulations, i.e. a 4-taps discrete channel with power loss $[0,-1,-3,-9][d B]$ and delay profile $[0,1,2,3] \mu s$ , (which corresponds to a urban type of scenario), a carrier frequency of $2.4 \mathrm{GHz}$ and a bandwidth $1 / T_{s}=1 \mathrm{MHz}$.

Simulations for three different speeds, $30 \mathrm{~km} / \mathrm{h}, 150 \mathrm{~km} / \mathrm{h}$ and $300 \mathrm{~km} / \mathrm{h}$ have been performed for an SNR equal to $20 \mathrm{~dB}$ (see Fig. 11). For reference, the performance of the algorithm is given by using the ideal channel state information (CSI). It is observed that our algorithm is far more robust to mobility than the literature algorithm proposed in [9]. This is logical since our algorithm has been devised to handle the high mobility, which is not the case of [9]. But to the best of our knowledge, we do not know an algorithm which performs channel and CFO estimation together with data detection in the presence of very high mobility. 


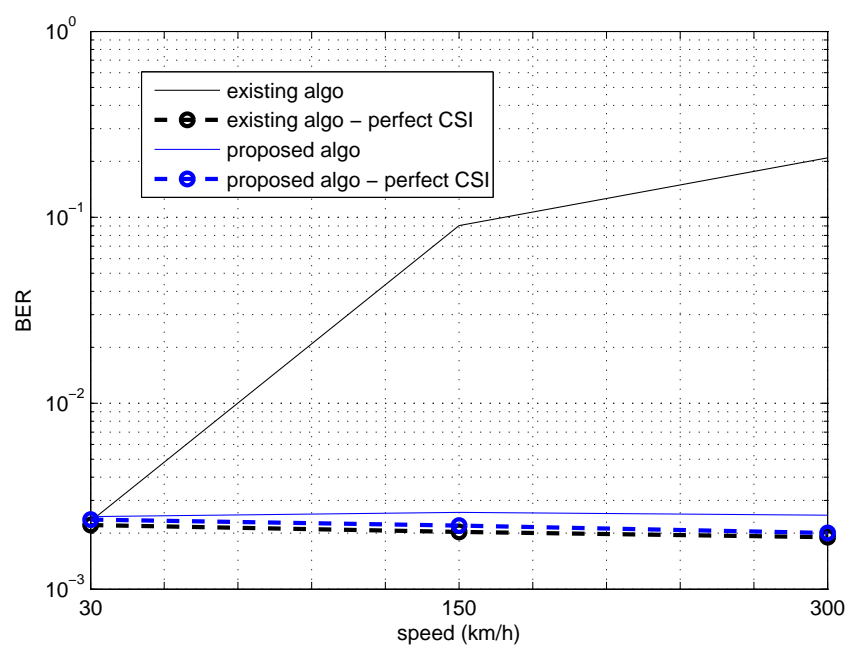

Fig. 11. BER performance at $E_{b} / N_{0}=20 \mathrm{~dB}$ - comparison with the algorithm of [9] for different speeds

\section{CONCLUSION}

In this paper, a new algorithm which jointly estimates path Complex Gains (CG) and Carrier Frequency Offset (CFO) in the presence of very high mobility has been presented. The algorithm is based on the EM algorithm. Within one OFDM symbol, each time-varying CG is approximated by a Basis Expansion Model (BEM) representation. The dynamics of the BEM coefficients are modeled by first-order auto-regressive processes. The algorithm operates in two steps, an acquisition step (pilot OFDM symbols) and a tracking step. Moreover, we have derived a closed-form exact Hybrid Cramér-Rao Bound (HCRB) for joint CFO and CG estimation in the presence of very high mobility. In contrast to the existing $\mathrm{CRB}$ for the estimation of fast time-varying CGs, the calculated bound does not assume a BEM, and hence represents the true lower bound. Simulation results have shown that the CG estimation reaches the HCRB. We also show that the proposed EM-based algorithm is a computationally less demanding technique than the MAP algorithm, with quasi same performance. Simulations have also shown that our algorithm addresses the problem of high mobility more efficiently than an existing algorithm. The proposed algorithm is also quite robust to reasonable uncertainties on the delays and the doppler frequency.

\section{APPENDIX A}

\section{COMPUTATION OF THE $Q$ FUNCTION}

Taking the expectation with respect to conditioned on $\mathbf{y}$, given the current parameter estimate $\hat{\nu}^{(i)}$, and removing the terms that do not depend on $\nu$, we obtain from (18):

$Q\left(\nu, \hat{\nu}^{(i)}\right)=-\frac{1}{\sigma^{2}} \sum_{k=0}^{K-1} \operatorname{Tr}\left\{\mathbb{E}_{\mathbf{c} \mid \mathbf{y}, \hat{\nu}^{(i)}}\left[\left(\mathbf{y}_{k}-\mathbf{m}_{k}(\nu)\right)\left(\mathbf{y}_{k}-\mathbf{m}_{k}(\nu)\right)\right.\right.$
From (32), we find:

$$
\begin{array}{r}
Q\left(\nu, \hat{\nu}^{(i)}\right)=-\frac{1}{\sigma^{2}} \sum_{k=0}^{K-1} \operatorname{Tr}\left\{\mathbf{y}_{k} \mathbf{y}_{k}^{H}+\mathcal{K}_{k}(\nu) \mathbf{P}_{k \mid K}^{(i)} \mathcal{K}_{k}^{H}(\nu)\right. \\
\left.-\mathbf{y}_{k} \hat{\mathbf{c}}_{k \mid K}^{(i)} \mathcal{K}_{k}^{H}(\nu)-\mathcal{K}_{k}(\nu) \hat{\mathbf{c}}_{k \mid K}^{(i)} \mathbf{y}_{k}^{H}\right\}
\end{array}
$$

where $\mathbf{P}_{k \mid K}^{(i)} \stackrel{\text { def }}{=} \mathbb{E}_{\mathbf{c} \mid \mathbf{y}, \hat{\nu}^{(i)}}\left[\mathbf{c}_{k} \mathbf{c}_{k}^{H}\right]$ and $\hat{\mathbf{c}}_{k \mid K}^{(i)} \stackrel{\text { def }}{=} \mathbb{E}_{\mathbf{c} \mid \mathbf{y}, \hat{\nu}^{(i)}}\left[\mathbf{c}_{k}\right]$. Let us define:

$$
\begin{aligned}
\mathbf{S}_{k \mid K}^{(i)} & \stackrel{\text { def }}{=} \mathbb{E}_{\mathbf{c} \mid \mathbf{y}, \hat{\nu}^{(i)}}\left[\left(\mathbf{c}_{k}-\hat{\mathbf{c}}_{k \mid K}^{(i)}\right)\left(\mathbf{c}_{k}-\hat{\mathbf{c}}_{k \mid K}^{(i)}\right)^{H}\right] \\
& =\mathbf{P}_{k \mid K}^{(i)}-\hat{\mathbf{c}}_{k \mid K}^{(i)} \hat{\mathbf{c}}_{k \mid K}^{(i){ }^{H}}
\end{aligned}
$$

Then, by using (34) in (33), we find (21).

\section{APPENDIX B}

\section{COMPUTATION OF THE HCRB}

In this appendix, we provide the main steps leading to the computation of the HCRB. First, we rearrange the system model given in (1) as follows:

$$
\mathbf{y}_{k}=\mathcal{H}_{k}(\nu) \cdot \boldsymbol{\alpha}_{k}+\mathbf{w}_{k}
$$

Let us define $\boldsymbol{\Omega}(\nu)=\left[e^{j 2 \pi \frac{0 \nu}{N}}, \ldots, e^{j 2 \pi \frac{(N-1) \nu}{N}}\right]^{T}$. The $N \times$ $L N$ matrix $\mathcal{H}_{k}(\nu)$ is defined as follows :

$$
\mathcal{H}_{k}(\nu)=\mathcal{W} \cdot \operatorname{diag}\{\Omega(\nu)\} \cdot \mathcal{L}_{k}
$$

with the $N \times L N$ matrix $\mathcal{L}_{k}$ given by:

$\left[\mathcal{L}_{k}\right]_{p, q}= \begin{cases}\boldsymbol{w}_{p}^{H} \operatorname{diag}\left\{\mathbf{x}_{k}\right\} \mathbf{f}_{l} & \text { if } q=p+l N, l=0 \ldots L-1 \\ 0 & \text { else }\end{cases}$

The $N \times N$ matrix $\mathcal{W}$ is the Fourier matrix $\left([\mathcal{W}]_{n, p}=\frac{1}{\sqrt{N}} e^{-j 2 \pi \frac{n p}{N}}\right)$ and vector $\boldsymbol{w}_{p}$ is the $p$ th column of $\mathcal{W}$.

1) Computation of: $\mathbb{E}_{\boldsymbol{\alpha}}[\mathbf{F i}(\boldsymbol{\alpha}, \nu)]$

First, we partition the matrix $\mathbf{F i}(\boldsymbol{\alpha}, \nu)$ as follows:

$$
\mathbf{F i}(\boldsymbol{\alpha}, \nu)=\left[\begin{array}{ll}
\mathbf{F i}_{11}(\boldsymbol{\alpha}, \nu) & \mathbf{F i}_{12}(\boldsymbol{\alpha}, \nu) \\
\mathbf{F i}_{21}(\boldsymbol{\alpha}, \nu) & \mathrm{Fi}_{22}(\boldsymbol{\alpha}, \nu)
\end{array}\right]
$$

where the submatrices are defined as follows:

$$
\begin{aligned}
& \mathbf{F i}_{11(\boldsymbol{\alpha}, \nu)}=\mathbb{E}_{\mathbf{y} \mid \boldsymbol{\alpha}, \nu}\left[-\left.\Delta_{\boldsymbol{\alpha}}^{\boldsymbol{\alpha}} \ln (p(\mathbf{y} \mid \boldsymbol{\alpha}, \nu))\right|_{\nu}\right] \\
& \mathbf{F i}_{12}(\boldsymbol{\alpha}, \nu)=\mathbb{E}_{\mathbf{y} \mid \boldsymbol{\alpha}, \nu}\left[-\left.\Delta_{\boldsymbol{\alpha}}^{\nu} \ln (p(\mathbf{y} \mid \boldsymbol{\alpha}, \nu))\right|_{\nu}\right] \\
& \mathbf{F i}_{21}(\boldsymbol{\alpha}, \nu)=\mathbb{E}_{\mathbf{y} \mid \boldsymbol{\alpha}, \nu}\left[-\left.\Delta_{\nu}^{\boldsymbol{\alpha}} \ln (p(\mathbf{y} \mid \boldsymbol{\alpha}, \nu))\right|_{\nu}\right] \\
& \mathrm{Fi}_{22}(\boldsymbol{\alpha}, \nu)=\mathbb{E}_{\mathbf{y} \mid \boldsymbol{\alpha}, \nu}\left[-\left.\Delta_{\nu}^{\nu} \ln (p(\mathbf{y} \mid \boldsymbol{\alpha}, \nu))\right|_{\nu}\right]
\end{aligned}
$$

Computation of $\mathbb{E}_{\boldsymbol{\alpha}}\left[\mathbf{F i}_{11(\boldsymbol{\alpha}, \nu)}\right]$ : the observation model is presented in (35). Using the whiteness of the noise $\mathbf{w}=$ $\left[\mathbf{w}_{0}^{T}, \ldots, \mathbf{w}_{K-1}^{T}\right]^{T}$, we obtain:

$$
\Delta_{\boldsymbol{\alpha}}^{\boldsymbol{\alpha}} \ln \left(p(\mathbf{y} \mid \boldsymbol{\alpha}, \nu)=\sum_{k=0}^{K-1} \Delta_{\boldsymbol{\alpha}}^{\boldsymbol{\alpha}} \ln \left(p\left(\mathbf{y}_{k} \mid \boldsymbol{\alpha}_{k}, \nu\right)\right)\right.
$$

It is important to note that each term of the summation (38) is a $K L N \times K L N$ block diagonal matrix with only one nonzero $L N \times L N$ block matrix, namely:

]$_{\boldsymbol{\delta}_{\boldsymbol{\alpha}}^{\boldsymbol{\alpha}}} \ln (p(\mathbf{y} \mid \boldsymbol{\alpha}, \nu))_{[i(k), i(k)]}=\Delta_{\boldsymbol{\alpha}_{k}}^{\boldsymbol{\alpha}_{k}} \ln \left(p\left(\mathbf{y}_{k} \mid \mathbf{x}_{k}, \boldsymbol{\alpha}_{k}, \nu\right)\right)$ 
where $i(k)=k L N:(k+1) L N-1$ with $k \in[0, K-1]$. As a direct consequence, $\Delta_{\boldsymbol{\alpha}}^{\boldsymbol{\alpha}} \ln (p(\mathbf{y} \mid \boldsymbol{\alpha}, \nu))$ is a block diagonal matrix with the $k$ th diagonal block given by (39). Hence, we obtain:

$$
\mathbb{E}_{\boldsymbol{\alpha}}\left[\mathbf{F i}_{11(\boldsymbol{\alpha}, \nu)}\right]=\operatorname{blkdiag}\left\{\mathcal{J}_{0(\nu)}, \mathcal{J}_{1(\nu)}, \ldots, \mathcal{J}_{K-1}(\nu)\right\}
$$

where $\mathcal{J}_{k}(\nu)$ is a $L N \times L N$ matrix defined as:

$$
\mathcal{J}_{k}(\nu)=\mathbb{E}_{\mathbf{y}, \boldsymbol{\alpha} \mid \nu}\left[-\Delta_{\boldsymbol{\alpha}_{k}}^{\boldsymbol{\alpha}_{k}} \ln \left(\left.p\left(\mathbf{y}_{k} \mid \boldsymbol{\alpha}_{k}, \nu\right)\right|_{\nu}\right)\right]
$$

It remains to compute the log-likelihood function in (40). The vector $\mathbf{y}_{k}$ for given $\boldsymbol{\alpha}_{k}$ is a complex Gaussian vector with mean vector $\mathbf{m}_{k}(\nu)=\mathcal{H}_{k}(\nu) \boldsymbol{\alpha}_{k}$ and covariance matrix $\sigma^{2} \mathbf{I}_{N}$.

Thus, $p\left(\mathbf{y}_{k} \mid \boldsymbol{\alpha}_{k}, \nu\right)$ is defined as:

$$
p\left(\mathbf{y}_{k} \mid \boldsymbol{\alpha}_{k}, \nu\right)=C e^{-\frac{1}{\sigma^{2}}\left(\mathbf{y}_{k}-\mathbf{m}_{k}(\nu)\right)^{H}\left(\mathbf{y}_{k}-\mathbf{m}_{k}(\nu)\right)}
$$

where $C$ is a constant. By taking the second derivative of the natural logarithm $\ln$ of (41) with respect to $\boldsymbol{\alpha}_{k}$, we simply obtain:

$$
\mathcal{J}_{k(\nu)}=\frac{1}{\sigma^{2}} \boldsymbol{H}_{k}^{H}(\nu) \mathcal{H}_{k}(\nu)
$$

Computation of $\mathbb{E}_{\boldsymbol{\alpha}}\left[\mathrm{Fi}_{22}(\boldsymbol{\alpha}, \nu)\right]:$ As for the computation of

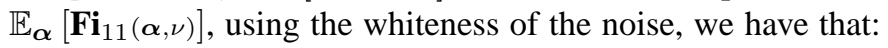

$$
\Delta_{\nu}^{\nu} \ln (p(\mathbf{y} \mid \boldsymbol{\alpha}, \nu))=\sum_{k=0}^{K-1} \Delta_{\nu}^{\nu} \ln \left(p\left(\mathbf{y}_{k} \mid \boldsymbol{\alpha}_{k}, \nu\right)\right)
$$

From (41), it follows that:

$$
\begin{aligned}
-\Delta_{\nu}^{\nu} \ln \left(p\left(\mathbf{y}_{k} \mid \boldsymbol{\alpha}_{k}, \nu\right)\right)=\frac{1}{\sigma^{2}}\left(-\mathbf{y}_{k}^{H} \Delta_{\nu}^{\nu}\left(\mathbf{m}_{k}(\nu)\right)\right. \\
\left.-\Delta_{\nu}^{\nu}\left(\mathbf{m}_{k}^{H}(\nu)\right) \mathbf{y}_{k}+\Delta_{\nu}^{\nu}\left(\mathbf{m}_{k}^{H}(\nu) \mathbf{m}_{k}(\nu)\right)\right)
\end{aligned}
$$

Then, we compute the expectation with respect to $\mathbf{y}$, yielding:

$$
\begin{array}{r}
\mathbb{E}_{\mathbf{y} \mid \boldsymbol{\alpha}, \nu}\left[-\Delta_{\nu}^{\nu} \ln \left(p\left(\mathbf{y}_{k} \mid \boldsymbol{\alpha}_{k}, \nu\right)\right)\right]=\frac{1}{\sigma^{2}}\left(-\mathbf{m}_{k}^{H} \Delta_{\nu}^{\nu}\left(\mathbf{m}_{k}(\nu)\right)\right. \\
\left.-\Delta_{\nu}^{\nu}\left(\mathbf{m}_{k}^{H}(\nu)\right) \mathbf{m}_{k}+\Delta_{\nu}^{\nu}\left(\mathbf{m}_{k}^{H}(\nu) \mathbf{m}_{k}(\nu)\right)\right),
\end{array}
$$

since $\mathbf{m}_{k}(\nu)=\mathcal{H}_{k}(\nu) \boldsymbol{\alpha}_{k}$ is the mean of $\mathbf{y}_{k}$. Using $\Delta_{\nu}^{\nu}(\mathbf{A}(\nu) \mathbf{B}(\nu))=\Delta_{\nu}^{\nu}(\mathbf{A}(\nu)) \mathbf{B}(\nu)+\mathbf{A}(\nu) \Delta_{\nu}^{\nu}(\mathbf{B}(\nu))+$ $2 \nabla_{\nu} \mathbf{A}(\nu) \nabla_{\nu} \mathbf{B}(\nu)$ with $\mathbf{A}(\nu)$ and $\mathbf{B}(\nu)$ some matrices depending on $\nu$, we obtain:

$$
\mathbb{E}_{\mathbf{y} \mid \boldsymbol{\alpha}, \nu}\left[-\left.\Delta_{\nu}^{\nu} \ln \left(p\left(\mathbf{y}_{k} \mid \boldsymbol{\alpha}_{k}, \nu\right)\right)\right|_{\nu}\right]=2 \boldsymbol{\alpha}_{k}^{H} \mathcal{J}_{k}^{\prime}(\nu) \boldsymbol{\alpha}_{k}
$$

where $\mathcal{J}_{k}^{\prime}(\nu)$ is a $L N \times L N$ matrix defined as:

$$
\mathcal{J}_{k}^{\prime}(\nu)=\frac{1}{\sigma^{2}} \mathcal{H}_{k}^{\prime H}(\nu) \mathcal{H}_{k}^{\prime}(\nu)
$$

where $\mathcal{H}_{k}^{\prime}(\nu) \stackrel{\text { def }}{=} \nabla_{\nu}\left(\mathcal{H}_{k}(\nu)\right)$ is obtained by replacing $\boldsymbol{\Omega}(\nu)$ with $\Omega^{\prime}(\nu)$ in (36), with:

$$
\boldsymbol{\Omega}^{\prime}(\nu)=\left[j 2 \pi \frac{0}{N} e^{j 2 \pi \frac{0 \nu}{N}}, \ldots, j 2 \pi \frac{(N-1)}{N} e^{j 2 \pi \frac{(N-1) \nu}{N}}\right]^{T}
$$

Then, computing $\mathbb{E}_{\boldsymbol{\alpha}}\left[\boldsymbol{\alpha}_{k}^{H} \mathcal{J}_{k}^{\prime}(\nu) \boldsymbol{\alpha}_{k}\right]$ yields, after short derivations, the sum of all the elements of the $L N \times L N$ matrix $\left(\mathbf{R}_{\alpha}^{(0)}{ }^{T} \circ \mathcal{J}_{k}^{\prime}(\nu)\right)$, that can be written as :

$$
\mathbb{E}_{\boldsymbol{\alpha}}\left[\boldsymbol{\alpha}_{k}^{H} \mathcal{J}_{k}^{\prime}(\nu) \boldsymbol{\alpha}_{k}\right]=\operatorname{Tr}\left\{\mathbf{R}_{\boldsymbol{\alpha}}^{(0)} \mathcal{J}_{k}^{\prime}(\nu)\right\}
$$

where $\mathbf{R}_{\boldsymbol{\alpha}}^{(0)} \stackrel{\text { def }}{=} \mathbb{E}\left[\boldsymbol{\alpha}_{k} \boldsymbol{\alpha}_{k}^{H}\right]$ is given by:

$$
\mathbf{R}_{\boldsymbol{\alpha}}^{(0)}{ }_{[i(l), i(l)]}=\mathbf{R}_{\boldsymbol{\alpha}_{l}}^{(0)} \text { for } l \in[0, L-1]
$$

with $i(l)=l N:(l+1) N-1$ and $\mathbf{R}_{\boldsymbol{\alpha}_{l}}^{(p)}$ defined in (5). Collecting all the obtained results, we finally get:

$$
\mathbb{E}_{\boldsymbol{\alpha}}\left[\mathrm{Fi}_{22}(\boldsymbol{\alpha}, \nu)\right]=2 \sum_{k=0}^{K-1} \operatorname{Tr}\left\{\mathbf{R}_{\boldsymbol{\alpha}}^{(0)} \mathcal{J}_{k}^{\prime}(\nu)\right\}
$$

Then, it is easily shown, by following a similar reasoning as above, that $\mathbb{E}_{\boldsymbol{\alpha}}\left[\mathbf{F i}_{12}(\boldsymbol{\alpha}, \nu)\right]=\mathbb{E}_{\boldsymbol{\alpha}}\left[\mathbf{F i}_{21}(\boldsymbol{\alpha}, \nu)\right]=0$.

2) Computation of $\mathbb{E}_{\boldsymbol{\alpha}}\left[-\Delta_{\boldsymbol{\alpha}}^{\boldsymbol{\alpha}} \ln (p(\boldsymbol{\alpha}))\right]: \boldsymbol{\alpha}$ is a complex Gaussian vector with zero mean and covariance matrix $\mathbf{R}_{\alpha}$ of size $K L N \times K L N$ defined as:

$$
\mathbf{R}_{\boldsymbol{\alpha}\left[i(l, p), i\left(l, p^{\prime}\right)\right]}=\mathbf{R}_{\boldsymbol{\alpha}_{l}}^{\left(p-p^{\prime}\right)} \text { for } l \in[0, L-1] p, p^{\prime} \in[0, K-1]
$$

where $i(l, p)=l N+p L N:(l+1) N-1+p L N$ and $\mathbf{R}_{\boldsymbol{\alpha}_{l}}^{(p)}$ is the correlation matrix of $\boldsymbol{\alpha}_{l, k}$ defined in (5). Thus, the probability density function $p(\boldsymbol{\alpha})$ is defined as:

$$
p(\boldsymbol{\alpha})=\frac{1}{\left|\pi \mathbf{R}_{\boldsymbol{\alpha}}\right|} e^{-\boldsymbol{\alpha}^{H} \mathbf{R}_{\alpha}^{-1} \boldsymbol{\alpha}}
$$

Taking the second derivative of the natural logarithm of (46) with respect to $\boldsymbol{\alpha}$ and making the expectation over $\boldsymbol{\alpha}$, we simply obtain that:

$$
\mathbb{E}_{\boldsymbol{\alpha}}\left[-\Delta_{\boldsymbol{\alpha}}^{\boldsymbol{\alpha}} \ln (p(\boldsymbol{\alpha}))\right]=\mathbf{R}_{\boldsymbol{\alpha}}^{-1}
$$

Collecting all those results yields the expression of the HCRB (29).

\section{REFERENCES}

[1] T. Pollet, M. V. Bladel, and M. Moeneclaey, "BER Sensitivity of OFDM Systems to Carrier Frequency Offset and Wiener Phase Noise," IEEE Trans. Commun., vol. 43, no. 2/3/4, pp. 191 - 193, February/March/April 1995.

[2] H. Steendam and M. Moeneclaey, "Sensitivity of Orthogonal FrequencyDivision Multiplexed Systems to Carrier and Clock Synchronisation Errors," Signal Processing, vol. 80, pp. 1217-1229, 2000.

[3] M. Ghogho and A. Swami, "Training Design for Multipath Channel and Frequency-Offset Estimation in MIMO Systems," IEEE Trans. on Signal Processing, vol. 54, 2006.

[4] V. Digalakis, J. Rohlicek, and M. Ostendorf, "ML estimation of a stochastic linear system with the EM algorithm and its application to speech recognition," IEEE Trans. Speech Audio Processing, vol. 1, pp. 431-442, 1993.

[5] Y. Xie and C. Georghiades, "Two em-type channel estimation algorithms for ofdm with transmitter diversity," Communications, IEEE Transactions on, vol. 51, no. 1, pp. $106-115$, jan 2003.

[6] J.-H. Lee, J. C. Han, and S.-C. Kim, "Joint carrier frequency synchronization and channel estimation for OFDM systems via the EM algorithm," IEEE Trans. Veh. Technol., vol. 55, no. 1, pp. 167 - 172, jan. 2006.

[7] S. Salari, M. Ardebilipour, and M. Ahmadian, "Joint maximumlikelihood frequency offset and channel estimation for multiple-input multiple-output-orthogonal frequency-division multiplexing systems," Communications, IET, vol. 2, no. 8, pp. 1069 -1076, sep. 2008.

[8] H. Abeida, J.-M. Brossier, L. Ros, and J. Vilà-Valls, "An EM algorithm for path delay and complex gain estimation of a slowly varying fading channel for CPM signals," in Proc. of the IEEE Globecom, Hawai, USA, 2009.

[9] T. Roman, M. Enescu, and V. Koivunen, "Joint Time-Domain Tracking of Channel and Frequency Offsets for MIMO OFDM Systems," Wireless Personal Communications, vol. 31, pp. 181-200, 2004. 
[10] M. Raghavendra, S. Bhashyam, and K. Giridhar, "Exploiting hopping pilots for parametric channel estimation in OFDM systems," IEEE Signal Process. Lett., vol. 12, no. 11, pp. 737 - 740, nov. 2005.

[11] O. Simeone, Y. Bar-Ness, and U. Spagnolini, "Pilot-based channel estimation for OFDM systems by tracking the delay-subspace," IEEE Trans. Wireless Commun., vol. 3, no. 1, pp. 315 - 325, jan. 2004.

[12] E. Simon, L. Ros, and K. Raoof, "Synchronization over Rapidly Timevarying Multipath Channel for CDMA Downlink RAKE Receivers in Time-Division Mode," IEEE Trans. Vehic. Techno., vol. 56, 2007.

[13] H. Hijazi and L. Ros, "Polynomial estimation of time-varying multi-path gains with intercarrier interference mitigation in OFDM systems," IEEE Trans. Vehic. Techno., vol. 57, 2008.

[14] B. Yang, K. B. Letaief, R. S. Cheng, and Z. Cao, "Channel estimation for OFDM transmisson in mutipath fading channels based on parametric channel modeling," IEEE Trans. Commun., vol. 49, pp. 467-479, 2001.

[15] H. Hijazi and L. Ros, "Joint Data QR-Detection and Kalman Estimation for OFDM Time-varying Rayleigh Channel Complex Gains," IEEE Trans. Comm., vol. 58, pp. 170-178, 2010.

[16] O. Besson and P. Stoica, "On parameter estimation of mimo flat-fading channels with frequency offsets," IEEE Trans. Signal Process., vol. 51, no. 3, pp. $602-613$, mar. 2003.

[17] H. Hijazi and L. Ros, "Analytical Analysis of Bayesian Cramer-Rao Bound for Dynamical Rayleigh Channel Complex Gains Estimation in OFDM System," IEEE Trans. Signal Process., vol. 57, 2009.

[18] Z. Tang, R. C. Cannizzaro, G. Leus, and P. Banelli, "Pilot-assisted timevarying channel estimation for OFDM systems," IEEE Trans. Signal Process., vol. 55, pp. 2226-2238, 2007.

[19] J.-G. Kim and J.-T. Lim, "MAP-Based Channel Estimation for MIMOOFDM Over Fast Rayleigh Fading Channels," IEEE Trans. Vehic. Techno., vol. 57, 2008.

[20] W. C. Jakes, Microwave Mobile Communications. IEEE Press, 1983.

[21] G. Leus, "On the Estimation of Rapidly Time-Varying Channels," in Euro. Signal Process. Conf. (EUSIPCO), 2004.

[22] T. Whitworth, M. Ghogho, and D. McLernon, "Optimized training and basis expansion model parameters for doubly-selective channel estimation," Wireless Communications, IEEE Transactions on, vol. 8, no. 3, pp. $1490-1498$, march 2009.

[23] K. E. Baddour and N. C. Beaulieu, "Autoregressive modeling for fading channel simulation," IEEE Trans. Wireless Commun., vol. 4, pp. 16501662,2005

[24] J. A. Bilmes, A Gentle Tutorial of the EM Algorithm and its Application to Parameter Estimation for Gaussian Mixture and Hidden Markov Models. Berkeley CA, 94704 and Computer Science Division Department of Electrical Engineering and Computer Science U.C. Berkeley TR97021, 1998

[25] H. Nguyen-Le and T. Le-Ngoc, "Pilot-aided joint cfo and doublyselective channel estimation for ofdm transmissions," Broadcasting, IEEE Transactions on, vol. 56, no. 4, pp. $514-522$, dec. 2010.

[26] Y. Rockah and P. M. Schultheiss, "Array shape calibration with sources in unknown locations, Part I: Far-field sources," IEEE Trans. Acoust., Speech, Signal Process., vol. ASSP-35, pp. 286-299, 1987.

[27] H. Messer, "The hybrid Cramr-Rao lower bound-from practice to theory," in Proceedings of IEEE Workshop on Sensor Array and Multichannel Signal processing, 2006.

[28] J. Andrews, A. Ghosh, and R. Muhamed, Fundamentals of WIMAX, S. Rappaport, Ed. prentice Hall, 2007.

[29] I. Barhumi, G. Leus, and M. Moonen, "Optimal training design for MIMO OFDM systems in mobile wireless channels," IEEE Transactions on Signal Processing, vol. 51, no. 6, pp. 1615 - 1624, june 2003.

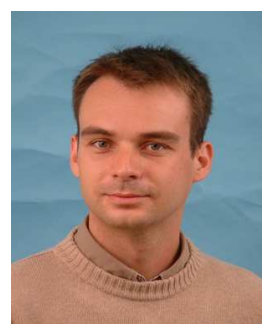

Eric P. Simon received the Masters degree in electronics engineering from the Superior School of Electronics (ESCPE), Lyon, France, in 1999, and the Ph.D. degree in signal processing and communications from the National Polytechnic Institute of Grenoble (INPG), France, in 2004. During 2005, he was a Teaching Assistant at the INPG and the following year he joined one of France Telecom $\mathrm{R} \& \mathrm{D}$ Laboratories as a Postdoctoral Fellow. He is currently an Associate Professor at the Institute of Electronics, Microelectronics and Nanotechnology (IEMN), TELICE (Telecommunications, Interference and Electromagnetic Compatibility) Group, University of Lille, France. His main research interests are in mobile communications and carrier and symbol synchronization.

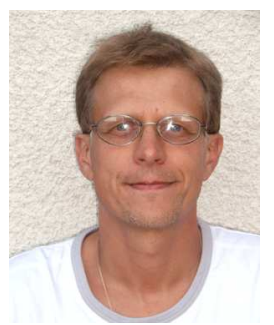

Laurent Ros received the degree in electrical engineering from the "Ecole Supérieure d'Electricité" (Supélec), Paris, France, in 1992 and the Ph.D. degree in signal processing and communications from Grenoble Institute of Technology (GrenobleINP), Grenoble, France, in 2001. From 1993 to 1995, he was with France-Telecom R\&D center, Lannion, France, where he worked in the area of very low frequency transmissions for submarine applications, in collaboration with Direction of Naval Construction, Toulon, France. From 1995 to 1999, he was a Research and Development team manager at Sodielec, Millau, France, where he worked in the design of digital modems and audio codecs for telecommunication applications. Since 1999, he has joined the Gipsalaboratory/DIS (ex "Laboratory of Image and Signal") and Grenoble-INP where he is currently an Associate Professor. His general research interests include statistical signal processing, synchronization and channel estimation problems for wireless communications.

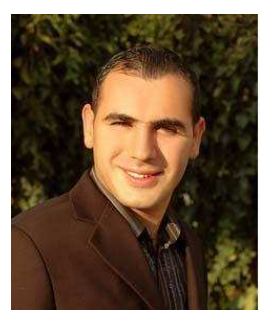

Hussein Hijazi is an assistant professor in the school of engineering at the Computer and Communication Engineering Department of Lebanese International University (LIU). He received his Diploma in computer and communications engineering in 2004 from the Lebanese University, Beirut, Lebanon. $\mathrm{He}$ received his M.S. and Ph.D. degrees in signal processing and communications in 2005 and 2008 respectively from the Institut Polytechnique de Grenoble (Grenoble-INP) ,Grenoble, France. From September 2008 to August 2009, he was an assistant professor at the engineering school ecole dingenieurs pour lenergie, leau et lenvironnement (ENSE3) of Grenoble-INP. From September 2009 to December 2009, he was a Research engineer in telecommunications at the IEMN laboratory of university of Lille 1. From December 2009 to December 2010, he was a Post-doctoral researcher at France Telecom-orange Labs, RD center, Meylan, France. His research interests are in the areas of signal processing and communications, including synchronisation, channel estimation and equalization problems for wireless digital communications and cooperative communications for wireless sensor network. Dr. Hijazi serves as a reviewer for several international journals and conferences including IEEE transactions on Signal Processing, IEEE transactions on communications, IEEE transactions on Wireless Communications, IEEE transactions on Vehicular Technology, and EURASIP Journal on Wireless Communications and Networking. 


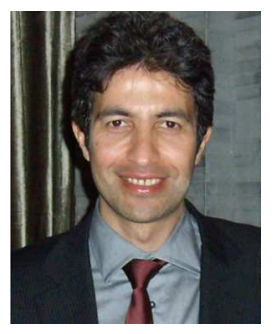

Mounir Ghogho received the M.S. degree in 1993 and the $\mathrm{PhD}$ degree in 1997 from the National Polytechnic Institute of Toulouse, France. He was an EPSRC Research Fellow with the University of Strathclyde, Glasgow, from September 1997 to November 2001. Since December 2001, he has been a faculty member with the school of Electronic and Electrical Engineering at the University of Leeds, where he is currently a Professor. He is also currently a Professor at the International University of Rabat in Morocco. He served as an Associate Editor of the IEEE Signal Processing Letters from 2001 to 2004 and the IEEE Transactions on Signal Processing from 2005 to 2008. He also served as a member of the IEEE Signal Processing Society SPCOM Technical Committee from 2005 to 2010 and is currently a member of the IEEE Signal Processing Society SPTM Technical Committee. He was the general co-chair of the eleventh IEEE workshop on Signal Processing for Advanced Wireless Communications (SPAWC'2010), the technical co-chair of the MIMO symposium of IWCMC 2007 and IWCMC 2008, and a technical area co-chair of Eusipco 2008, Eusipco 2009 and ISCCSP'05. He is the general chair of Eusipco2013. He was the guest co-editor of the EURASIP Journal on Wireless Communications and Networking special issue on "synchronization for wireless communications" and the Elsevier Physical Communication special issue on "advances in MIMO-OFDM." His research interests are in communication and sensor networks, radar imaging and signal processing. Prof. Ghogho was awarded a five-year Royal Academy of Engineering Research Fellowship in September 2000. 